Pacific

Journal of

Mathematics

\title{
SINGULAR FIBERS OF TWO-COLORED MAPS AND COBORDISM INVARIANTS
}

TAKAHIRO YAMAMOTO 


\title{
SINGULAR FIBERS OF TWO-COLORED MAPS AND COBORDISM INVARIANTS
}

\author{
TAKAHIRO YAMAMOTO
}

To my teacher Go-o Ishikawa, on the occasion of his 50-th birthday

\begin{abstract}
We introduce two-colored maps of $n$-manifolds into $\boldsymbol{m}$-manifolds, develop the theory of their singular fibers, and study these fibers for the case $\boldsymbol{m}=\mathbf{4}$ and $n=3,5$.
\end{abstract}

\section{Introduction}

Following the pioneering work of Thom [1954] on the cobordisms for embeddings of manifolds, Koschorke [1981] and Rimányi and Szúcs [1998] introduced the notion of cobordisms of singular maps with positive codimension. The codimension of a map $f: M \rightarrow N$ between manifolds is $\operatorname{dim} N-\operatorname{dim} M$. In particular, "universal singular maps" were constructed by using the Pontrjagin-Thom construction in [Rimányi and Szúcs 1998]. (Each singular map can be pulled back from the universal singular maps.) For the negative codimension case, Ikegami and Saeki [2003] defined and studied the cobordism group of Morse functions on surfaces. Then Kalmar [2007] generalized this result for fold maps of oriented 3-manifolds into the plane.

Saeki [2004] developed the theory of the fibers of smooth maps with negative codimension. For a smooth map $f: M \rightarrow N$, the fiber over $q \in N$ is a map germ along the inverse image

$$
f:\left(M, f^{-1}(q)\right) \rightarrow(N, q) .
$$

When $q \in N$ is a regular value of $f$, we call $f:\left(M, f^{-1}(q)\right) \rightarrow(N, q)$ a regular fiber; otherwise, we call it a singular fiber. In the negative codimension case, the inverse image is not a discrete set but a complex of positive dimension, and Saeki's book shows the topology of the fibers plays an essential role. He constructed the cochain complex of the fibers, and showed that the cohomology classes of this complex induce cobordism invariants of smooth maps. As an important example,

MSC2000: primary 57R45; secondary 57N13.

Keywords: colored map, Thom map, stable map, singular fiber, bordism, cobordism. 
he showed this: For each stable map of an orientable closed 4-manifold into a connected 3-manifold, the number of singular fibers of III ${ }^{12}$ type (as depicted in Figure 1) and the Euler number of the 4-manifold have the same parity. Saeki uses the symbol "III" ${ }^{8}$ instead of "III ${ }^{12 "}$. Note that the modulo two Euler number is one of the unoriented cobordism invariants of 4-manifolds; see [Milnor and Stasheff 1974] for details.

Saeki's Euler number formula was generalized for nonorientable 4-manifolds in [Yamamoto 2006a]. As an "integral lift" of the formula, Saeki and Yamamoto [2006] obtained the signature formula of oriented closed 4-manifolds.

Here, we consider the fibers of smooth maps from a global point of view. More precisely, we study the fibers of two-colored maps. Roughly speaking, a twocolored map is a map equipped with a two-color painting for the complements of the discriminant in the target; see Section 2 for details. We introduce an equivalence relation among the fibers of two-colored maps and the notion of two-colored cobordism between two-colored maps. We also develop the theory of fibers of two-colored maps, in which we construct the cochain complex of the fibers of twocolored maps by using the adjacencies of the fibers. We prove that cohomology classes of the cochain complex induce two-colored cobordism invariants of twocolored maps.

This paper is organized as follows. In Section 2, we give some fundamental definitions concerning two-colored maps. In Section 3, we construct the theory of fibers of two-colored maps. In Section 4, we study two-colored stable maps of $n$-manifolds into 4-manifolds for $n=3$ and $n=5$. As an application of our theory, we prove two theorems:

Theorem 1.1 [Yamamoto 2006a]. Let $N$ be a connected 3-manifold satisfying $H^{1}\left(N ; \mathbb{Z}_{2}\right)=0$, and let $f: M \rightarrow N$ be a stable map of a closed 4 -manifold. Then

$$
\begin{aligned}
\chi(M) \equiv\left|\widetilde{\mathrm{II}}^{2,2,2}(f)\right|+\left|\widetilde{\mathrm{II}}^{2,7}(f)\right| & +\left|\widetilde{\mathrm{II}}^{12}(f)\right|+\left|\widetilde{\mathrm{II}}_{o, A}^{13}(f)\right| \\
& +\left|\widetilde{\mathrm{III}}_{e, B}^{13}(f)\right|+\left|\widetilde{\mathrm{II}}^{25}(f)\right|+\left|\widetilde{\mathrm{II}}^{26}(f)\right| \quad(\bmod 2),
\end{aligned}
$$

where $\chi(M)$ is the Euler number of $M$ and $|\mathscr{F}(f)|$ is the number of singular fibers of type F for $f$, as depicted in Figure 1.

Theorem 1.2 [Szúcs 1986]. Let $N$ be a connected 3-manifold with $H^{1}\left(N ; \mathbb{Z}_{2}\right)=0$, and let $g: S \rightarrow N$ be a stable map of a closed surface. Then

$$
\chi(S) \equiv T(g)+\sum_{\begin{array}{c}
\text { Whitney umbrella } \\
\text { points } q
\end{array}} \operatorname{ind}(q ; g) \quad(\bmod 2),
$$

where $T(g)$ is the number of triple points of $g$ and ind $(q ; g) \in \mathbb{Z}_{2}$ is the index of the Whitney umbrella point $q \in N$ of $g$; see Section 4.1 for details. 


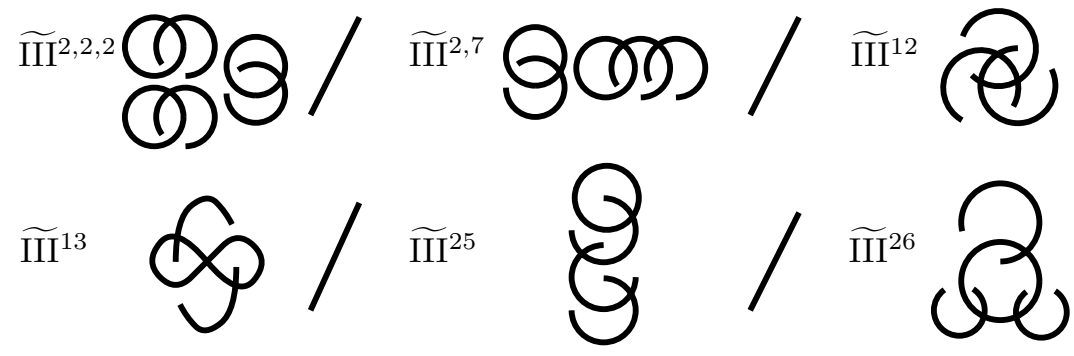

Figure 1. The singular fibers in the formula of Theorem 1.1.

Throughout this paper, assume that all manifolds are connected and that all manifolds and maps are of the class $C^{\infty}$. Call $p \in M$ a critical point of $f: M \rightarrow N$ if the rank of the Jacobi matrix of $f$ at $p$ is less than the dimension of the target; otherwise call it a regular point. (In this sense, there are no regular points if the dimension of the source is less than that of the target.) Denote the set of critical points of $f$ by $S(f)$. For a finite set $P$, let $|P|$ denote the number of elements in $P$. For a topological space $X$, the symbols $\operatorname{id}_{X}, \chi(X)$, and $\bar{X}$ respectively denote the identity map on $X$, the Euler number of $X$, and the topological closure of $X$.

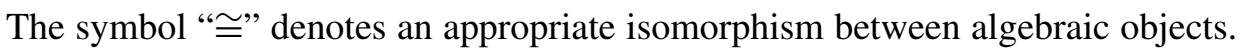

The paper is based on the author's doctoral thesis [Yamamoto 2006b]. Omitted proofs may be found there or in [Saeki 2004].

\section{Preparation}

Let $f: M \rightarrow N$ be a smooth map with $\operatorname{dim} M+1 \geq \operatorname{dim} N$.

Definition 2.1. The map $f$ is two-colorable if there exists a pair of disjoint nonempty open subsets $R$ and $B$ in $N \backslash f(S(f))$ such that

$$
N \backslash f(S(f))=R \cup B \quad \text { and } \quad \bar{R} \cap \bar{B}=\partial R=\partial B=f(S(f)) .
$$

If $f$ has no critical values, it is also two-colorable. Call the pair $(R, B)$ satisfying the condition (2-1) a two-color decomposition or a coloring of $f$.

Figure 2 shows examples of two-colored maps, where the shadowed regions indicate $R$.

Proposition 2.2. $f: M \rightarrow N$ is two-colorable if and only if $f_{*}[S(f)]=0 \in$ $H_{p-1}^{c}\left(N ; \mathbb{Z}_{2}\right) \cong H^{1}\left(N ; \mathbb{Z}_{2}\right)$, where $[S(f)]$ denotes the homology class represented by $S(f)$.

Proof. Fix $q_{0} \in N \backslash f(S(f))$. For each $q \in N \backslash f(S(f))$, choose a regular curve $\gamma$ connecting $q_{0}$ with $q$ and intersecting $f(S(f))$ transversely at finitely many points. We prove that the parity of the intersection number of $\gamma$ and $f(S(f))$ does 


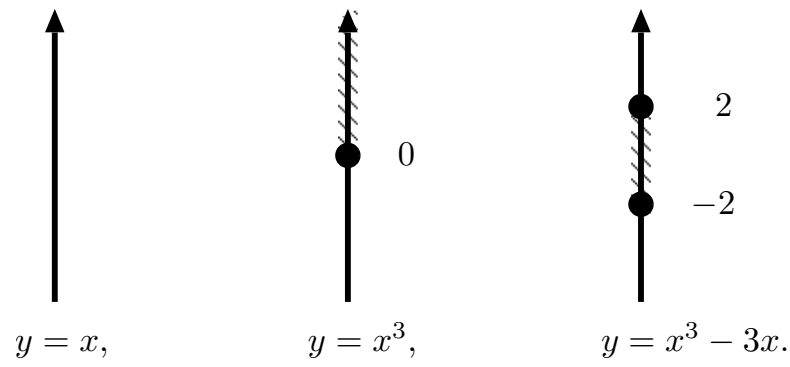

Figure 2. Examples of two-colored maps.

not depend on the choice of $\gamma$. For another curve $\gamma^{\prime}$ connecting $q_{0}$ with $q$ and intersecting $f(S(f))$ transversely at finitely many points, we see

$$
\#(\gamma \cap f(S(f)))+\#\left(\gamma^{\prime}\right) \cap f(S(f)) \equiv\left[\gamma \cup \gamma^{\prime}\right] \cdot[f(S(f))] \equiv 0 \quad(\bmod 2),
$$

where $\left[\gamma \cup \gamma^{\prime}\right] \cdot[f(S(f))]$ is the modulo two intersection number of the homology classes represented by a circle $\gamma \cup \gamma^{\prime}$ and by $f(S(f))$. Put

$$
\begin{aligned}
& R=\{q \in N \backslash f(S(f)): \#(\gamma \cap f(S(f))) \text { is even }\} \\
& B=\{q \in N \backslash f(S(f)): \#(\gamma \cap f(S(f))) \text { is odd }\} .
\end{aligned}
$$

It is obvious that $R$ and $B$ are disjoint nonempty open subsets. Conversely, by the assumption that $f$ is two-colorable for each circle intersecting $f(S(f))$ transversely at finite points, the intersection number of the circle and $f(S(f))$ is always even. Now Poincaré duality implies that $f_{*}[S(f)]=0$.

Note that $f: M \rightarrow N$ is two-colorable if $N=\mathbb{R}^{n}$ or $M$ is orientable.

Call the pair $(f,(R, B))$ of a two-colorable map $f$ and a coloring $(R, B)$ of $f$ a two-colored map or a colored map. For a two-colored map $(f,(R, B))$, there exists another two-colored map $(f,(\tilde{R}, \tilde{B}))$ where $\tilde{R}=B$ and $\tilde{B}=R$. We regard these to be distinct two-colored maps. Call the latter two-colored map the two-colored conjugate of $(f,(R, B))$.

Definition 2.3. Let $\left(f_{i},\left(R_{i}, B_{i}\right)\right): M_{i} \rightarrow N_{i}$ be two-colored maps and $q_{i} \in N_{i}$ for $i=0,1$. Say the fibers over $q_{0}$ and $q_{1}$ are $C^{\infty}$ equivalent (or $C^{0}$ equivalent) if there exist open neighborhoods $U_{i}$ of $q_{i} \in N_{i}$ for $i=0,1$ and diffeomorphism (or homeomorphism) germs $\Phi:\left(f^{-1}\left(U_{0}\right), f^{-1}\left(q_{0}\right)\right) \rightarrow\left(f^{-1}\left(U_{1}\right), f^{-1}\left(q_{1}\right)\right)$ and $\varphi:\left(U_{0}, q_{0}\right) \rightarrow\left(U_{1}, q_{1}\right)$ such that the following diagram is commutative:

$$
\begin{array}{ccc}
\left(f_{0}^{-1}\left(U_{0}\right), f_{0}^{-1}\left(q_{0}\right)\right) & \stackrel{\Phi}{\longrightarrow} & \left(f_{1}^{-1}\left(U_{1}\right), f_{1}^{-1}\left(q_{1}\right)\right) \\
f_{0} \downarrow & & \downarrow f_{1} \\
\left(U_{0}, q_{0}\right) & \stackrel{\varphi}{\longrightarrow} & \left(U_{1}, q_{1}\right) .
\end{array}
$$

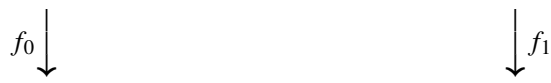


Denote the $C^{\infty}$ equivalence (or $C^{0}$ equivalence) relation by $\rho^{\infty}$ (or $\rho^{0}$ ).

Also, say the fibers over $q_{0}$ and $q_{1}$ are two-colored $C^{\infty}$ equivalent (or twocolored $C^{0}$ equivalent) if they are $C^{\infty}$ equivalent (or $C^{0}$ equivalent) and the diffeomorphism (or homeomorphism) germ $\varphi:\left(U_{0}, q_{0}\right) \rightarrow\left(U_{1}, q_{1}\right)$ in diagram (2-2) satisfies $\varphi\left(U_{0} \cap R_{0}\right)=U_{1} \cap R_{1}$. Denote the two-colored $C^{\infty}$ equivalence (or twocolored $C^{0}$ equivalence) relation by $c \rho^{\infty}\left(\right.$ or $\left.c \rho^{0}\right)$.

For a two-colored map $(f,(R, B)): M \rightarrow N$, there exists a point $q \in N$ such that the fiber of $(f,(R, B))$ over $q$ is not two-colored $C^{\infty}$ equivalent to the fiber of the two-colored conjugate of $(f,(R, B))$ over $q$; see Figure 5 and 6 . Call the latter fiber the two-colored conjugate of the original fiber.

For a two-colored Thom map ${ }^{1}(f,(R, B)): M \rightarrow N$ and a two-colored $C^{0}$ class $\mathscr{F}$, denote the set of points whose fibers are of type $\mathscr{F}$ in $N$ by $\mathscr{F}(f,(R, B))$.

Lemma 2.4. $\mathscr{F}(f,(R, B))$ is a $C^{0}$ submanifold of $N$ of constant codimension unless it is empty. This codimension does not depend on the choice of $(f,(R, B))$.

\section{Cochain complex of the singular fibers of two-colored maps}

We construct the theory of the fibers of proper $^{2}$ two-colored Thom maps.

To construct the universal complex of the singular fibers of two-colored maps, fix an integer $\ell \in \mathbb{Z}$. For $\ell$, introduce

(1) the set $c \tau$ of the fibers of two-colored Thom maps of codimension $\ell$, and

(2) an equivalence relation $c \rho$ among fibers in $c \tau$.

The set $c \tau$ and the equivalence $c \rho$ must each satisfy certain conditions.

The set $c \tau$ must be closed under adjacency relations and the two-colored conjugation. That is, if a fiber is in $c \tau$, so are all nearby fibers and its conjugate.

The equivalence $c \rho$ is weaker than the two-colored $C^{0}$ equivalence. Namely, each $c \rho$ class is a union of two-colored $C^{0}$ classes. This implies that for each proper two-colored Thom map $(f,(R, B))$ and each $c \rho$ class $\mathscr{F}, \mathscr{F}(f,(R, B))$ is a $C^{0}$ submanifold of constant codimension unless it is empty. The codimension of $\mathscr{F}$ is defined to be the codimension of $\mathscr{F}(f,(R, B))$. Denote it by $\kappa(\mathscr{F})$.

Also, $c \rho$ must satisfy the following condition.

Condition 3.1. Suppose $\left(f_{i},\left(R_{i}, B_{i}\right)\right): M_{i} \rightarrow N_{i}$ are proper two-colored Thom maps and $q_{i} \in N_{i}$ for $i=0,1$. Suppose the fibers over $q_{0}$ and $q_{1}$ are in $c \tau$ and are equivalent with respect to $c \rho$. There exist open neighborhoods $U_{i}$ of $q_{i}$ in

${ }^{1}$ A Thom map $f: M \rightarrow N$ is a map stratified with respect to the Whitney regular stratification of $M$ and $N$ so that it is a submersion on each stratum and satisfies certain regularity conditions, see [Gibson et al. 1976] for details. A two-colored Thom map is a Thom map equipped with a coloring.

${ }^{2}$ A continuous map is proper if for each compact subset, the inverse image is also compact. 
$N_{i}$ for $i=0,1$ and a homeomorphism $\varphi: U_{0} \rightarrow U_{1}$ satisfying $\varphi\left(q_{0}\right)=q_{1}$ and $\varphi\left(U_{0} \cap \mathscr{F}\left(f_{0},\left(R_{0}, B_{0}\right)\right)\right)=U_{1} \cap \mathscr{F}\left(f_{1},\left(R_{1}, B_{1}\right)\right)$ for each $c \rho$ class $\mathscr{F}$.

Call a proper two-colored Thom map $(f,(R, B)): M \rightarrow N$ a $c \tau-m a p$ if all of its fibers are in $c \tau$.

For each $\kappa \in \mathbb{Z}$, let $C^{\kappa}(c \tau, c \rho)$ be the formal $\mathbb{Z}_{2}$-vector space spanned by $c \rho$ classes of codimension $\kappa$ in $c \tau$. Note that $C^{\kappa}(c \tau, c \rho)$ may possibly contain infinitely many terms. If there are no such fibers, put $C^{\kappa}(c \tau, c \rho)=0$.

Define a $\mathbb{Z}_{2}$-linear map $\delta_{\kappa}: C^{\kappa}(c \tau, c \rho) \rightarrow C^{\kappa+1}(c \tau, c \rho)$ by

$$
\delta_{\kappa}(\mathscr{F})=\sum_{\kappa(\mathscr{G})=\kappa+1} n_{\mathscr{F}}(\mathscr{G}) \mathscr{G} .
$$

Here $n_{\mathscr{F}}(\mathscr{G}) \in \mathbb{Z}_{2}$ is the number modulo two of components of $\mathscr{F}(f,(R, B))$ that are locally adjacent to the component $\mathscr{G}(f,(R, B))$ for a $c \tau$-map $(f,(R, B))$ satisfying $\mathscr{G}(f,(R, B)) \neq \varnothing$. The coefficient $n_{\mathscr{F}}(\mathscr{G}) \in \mathbb{Z}_{2}$ is well defined by Condition 3.1.

Since $\delta_{\kappa+1} \circ \delta_{\kappa}=0$, we obtain a cochain complex

$$
\mathscr{C}(c \tau, c \rho)=\left(C^{\kappa}(c \tau, c \rho), \delta_{\kappa}\right)_{\kappa} .
$$

Call the resulting cochain complex the universal complex of the singular fibers for proper $c \tau$-maps of codimension $\ell$ with respect to the equivalence relation $c \rho$, and denote by $H^{\kappa}(c \tau, c \rho)$ its cohomology group in dimension $\kappa$.

Remark 3.2. Define a homomorphism

$$
\gamma_{\kappa}: C^{\kappa}(c \tau, c \rho) \rightarrow C^{\kappa}(c \tau, c \rho), \quad \mathscr{F} \mapsto \widetilde{\mathscr{F}},
$$

where $\widetilde{\mathscr{F}}$ denotes the $c \rho$ class represented by the fiber of the two-colored conjugate of $\mathscr{F}$. Note that $\gamma_{\kappa}$ is an involution, that is, $\gamma_{\kappa} \circ \gamma_{\kappa}=\operatorname{id}_{C^{\kappa}(c \tau, c \rho)}$, and $\gamma=\left\{\gamma_{\kappa}\right\}_{\kappa}$ is a cochain map. The quotient of this involution induces a certain universal complex of the singular fibers for proper $\tau$-maps of codimension $\ell$ with respect to the equivalence relation $\rho$ constructed in [Saeki 2004], where $\tau$ denotes the set of the fibers of Thom maps and $\rho$ is an equivalence relation among elements in $\tau$.

Definition 3.3. Let

$$
c=\sum_{\kappa(\mathscr{F})=\kappa} n_{\mathscr{F}} \mathscr{F}
$$

be a $\kappa$-dimensional cochain of $\mathscr{C}(c \tau, c \rho)$. For a $c \tau$-map $(f,(R, B)): M \rightarrow N$, denote by $c(f,(R, B))$ the set of points $q \in N$ such that the fiber over $q$ is in $\mathscr{F}$ with $n_{\mathscr{F}} \neq 0$. If $c$ is a cocycle, $c(f,(R, B))$ is a $\mathbb{Z}_{2}$-cycle of closed support of codimension $\kappa$ in $N$. In addition, if $M$ is closed and $\kappa>0$, then $c(f,(R, B))$ is a $\mathbb{Z}_{2}$-cycle in the usual sense. 
Lemma 3.4. Let $c$ and $c^{\prime}$ be $\kappa$-dimensional cocycles of $\mathscr{C}(c \tau, c \rho)$. If they are cohomologous, the $\mathbb{Z}_{2}$-cycles $c(f,(R, B))$ and $c^{\prime}(f,(R, B))$ are homologous in $N$ for each c $\tau$-map $(f,(R, B)): M \rightarrow N$.

Proof. There exists a $(\kappa-1)$-dimensional cochain $d$ of $\mathscr{C}(c \tau, c \rho)$ such that $c-c^{\prime}=$ $\delta_{\kappa-1} d$. Since $c(f)-c^{\prime}(f)=\partial d(f)$, the result follows.

Definition 3.5. Let $[c]$ be a $\kappa$-dimensional cohomology class of $\mathscr{C}(c \tau, c \rho)$. For a proper $c \tau$-map $(f,(R, B)): M \rightarrow N$, define $[c(f,(R, B))] \in H_{p-\kappa}^{c}\left(N, \mathbb{Z}_{2}\right)$ to be the homology class represented by the cycle $c(f,(R, B))$ of closed support. When $M$ is closed and $\kappa>0$, regard $[c(f,(R, B))]$ as an element of $H_{p-\kappa}\left(N, \mathbb{Z}_{2}\right)$.

Define

$$
\varphi_{(f,(R, B))}: H^{\kappa}(c \tau, c \rho) \rightarrow H^{\kappa}\left(N, \mathbb{Z}_{2}\right)
$$

by $\varphi_{(f,(R, B))}([c])=[c(f,(R, B))]^{*}$, where $[c(f,(R, B))]^{*} \in H^{\kappa}\left(N, \mathbb{Z}_{2}\right)$ is the Poincaré dual of $[c(f,(R, B))] \in H_{p-\kappa}^{c}\left(N, \mathbb{Z}_{2}\right)$. When $M$ is closed and $\kappa>0$, regard $\varphi_{(f,(R, B))}$ as a homomorphism into the cohomology group $H_{c}^{\kappa}\left(N, \mathbb{Z}_{2}\right)$ of compact support.

We now introduce the suspension of two-colored Thom maps.

Definition 3.6. For a proper two-colored Thom map $(f,(R, B)): M \rightarrow N$, consider

$$
f \times \mathrm{id}_{\mathbb{R}}: M \times \mathbb{R} \rightarrow N \times \mathbb{R} .
$$

Note that $S\left(f \times \operatorname{id}_{\mathbb{R}}\right)=S(f) \times \mathbb{R}$ and $f \times \operatorname{id}_{\mathbb{R}}(S(f) \times \mathbb{R})=f(S(f)) \times \mathbb{R}$. We obtain a two-colored map $\left(f \times \operatorname{id}_{\mathbb{R}},(R \times \mathbb{R}, B \times \mathbb{R})\right): M \times \mathbb{R} \rightarrow N \times \mathbb{R}$. Call $\left(f \times \operatorname{id}_{\mathbb{R}},(R \times \mathbb{R}, B \times \mathbb{R})\right)$ the suspension of $(f,(R, B))$. Similarly, call the fiber of $\left(f \times \operatorname{id}_{\mathbb{R}},(R \times \mathbb{R}, B \times \mathbb{R})\right)$ over $(q, 0) \in N \times \mathbb{R}$ the suspension of the fiber of $(f,(R, B))$ over $q \in N$.

In what follows, assume that $c \tau$ consists of certain fibers of proper two-colored Thom maps of $n$-manifolds into $p$-manifolds for a fixed dimension pair $p-n=\ell$. In this case, write $c \tau=c \tau(n, p)$. We consider $c \tau(n, p)$ and $c \tau(n+1, p+1)$ and their associated equivalence relations $c \rho_{n, p}$ and $c \rho_{n+1, p+1}$, respectively. In addition to Condition 3.1, impose

(1) the suspension of each fiber in $c \tau(n, p)$ is also in $c \tau(n+1, p+1)$, and

(2) if two fibers are equivalent with respect to $c \rho_{n, p}$, their suspensions are also equivalent with respect to $c \rho_{n+1, p+1}$.

For each $\kappa \in \mathbb{Z}$, the suspension induces

$$
s_{\kappa}: C^{\kappa}\left(c \tau(n+1, p+1), c \rho_{n+1, p+1}\right) \rightarrow C^{\kappa}\left(c \tau(n, p), c \rho_{n, p}\right),
$$

where $s_{\kappa}(\mathscr{F})$ is the (possibly infinite) sum of all $c \rho_{n, p}$ classes whose suspensions are in $\mathscr{F}$ of codimension $\kappa$. Note that $s_{\kappa}$ is a well-defined $\mathbb{Z}_{2}$-linear map. 
Lemma 3.7. The system of $\mathbb{Z}_{2}$-linear maps $\left\{s_{\kappa}\right\}$ defines the cochain map

$$
\left\{s_{\kappa}\right\}: \mathscr{C}\left(c \tau(n+1, p+1), c \rho_{n+1, p+1}\right) \rightarrow \mathscr{C}\left(c \tau(n, p), c \rho_{n, p}\right) .
$$

Let us introduce an equivalence relation between $c \tau$-maps.

Definition 3.8. Two $c \tau$-maps $\left(f_{i},\left(R_{i}, B_{i}\right)\right): M_{i} \rightarrow N$ for $i=0,1$ of closed manifolds into $N$ are $c \tau$-cobordant if there exist a compact manifold $W$ whose boundary is the disjoint union of $M_{0}$ and $M_{1}$ and a $c \tau$-map $(F,(R, B)): W \rightarrow N \times[0,1]$ such that $\left(f_{i},\left(R_{i}, B_{i}\right)\right)=\left.(F,(R, B))\right|_{M_{i}}: M_{i} \rightarrow N \times\{i\}$. Call the map $(F,(R, B))$ a $c \tau$-cobordism between $\left(f_{0},\left(R_{0}, B_{0}\right)\right)$ and $\left(f_{1},\left(R_{1}, B_{1}\right)\right)$.

Note that $c \tau$-cobordance is an equivalence relation among $c \tau$-maps into $N$. For a manifold $N$, denote by $\operatorname{Cob}_{c \tau}(N)$ the set of $c \tau$-cobordant equivalence classes among $c \tau$-maps of closed manifolds into $N$. We remark that $\operatorname{Cob}_{c \tau}(N)$ has no natural group structure.

Proposition 3.9. If two c $\tau$-maps $\left(f_{i},\left(R_{i}, B_{i}\right)\right): M_{i} \rightarrow N$ of closed manifolds into $N$ for $i=0,1$ are $c \tau$-cobordant, then for each $\kappa$

$$
\left.\varphi_{\left(f_{0},\left(R_{0}, B_{0}\right)\right)}\right|_{\operatorname{Im} s_{\kappa *}}=\left.\varphi_{\left(f_{1},\left(R_{1}, B_{1}\right)\right)}\right|_{\operatorname{Im} s_{\kappa *}}: \operatorname{Im} s_{\kappa_{*}} \rightarrow H^{\kappa}\left(N ; \mathbb{Z}_{2}\right),
$$

where $s_{\kappa_{*}}: H^{\kappa}\left(c \tau(n+1, p+1), c \rho_{n+1, p+1}\right) \rightarrow H^{\kappa}\left(c \tau(n, p), c \rho_{n, p}\right)$ denotes the homomorphism induced by the suspension.

Proof. Let $(F,(R, B)): W \rightarrow N \times[0,1]$ be a $c \tau$-cobordism between $\left(f_{0},\left(R_{0}, B_{0}\right)\right)$ and $\left(f_{1},\left(R_{1}, B_{1}\right)\right)$. Take from $\mathscr{C}\left(c \tau(n+1, p+1), c \rho_{n+1, p+1}\right)$ any $\kappa$-dimensional cocycle $c$, and put $\bar{c}=s_{\kappa}(c) \in C^{\kappa}\left(c \tau(n, p), c \rho_{n, p}\right)$. Then $0=\left(\delta_{\kappa} c\right)((F,(R, B)))=$ $\partial c((F,(R, B)))=\bar{c}\left(\left(f_{1},\left(R_{1}, B_{1}\right)\right)\right) \times\{1\}-\bar{c}\left(\left(f_{0},\left(R_{0}, B_{0}\right)\right)\right) \times\{0\}$, since $c$ is a cocycle.

Thus, for each $[c] \in H^{\kappa}\left(c \tau(n+1, p+1), c \rho_{n+1, p+1}\right)$, we obtain a map

$$
[c]: \operatorname{Cob}_{c \tau}(N) \rightarrow H^{\kappa}\left(N ; \mathbb{Z}_{2}\right), \quad(f,(R, B)) \mapsto \varphi_{(f,(R, B))}\left(\left[s_{\kappa *} c\right]\right) .
$$

Each element in $H^{\kappa}\left(c \tau(n+1, p+1), c \rho_{n+1, p+1}\right)$ induces a $c \tau(\kappa+n-p+1, \kappa+1)$ cobordism invariant among $c \tau(\kappa+n-p, \kappa)$-maps $(f,(R, B)): M^{\kappa+n-p} \rightarrow N^{\kappa}$, where $\kappa=1,2, \ldots, p$.

Remark 3.10. Assume $c \tau$ is big enough. Then, for each smooth map $f: M \rightarrow$ $\mathbb{R}^{n}$ of a closed manifold, define $\varphi_{f}$ by $\varphi_{(\widetilde{f},(\widetilde{R}, \widetilde{B}))}$, where $(\widetilde{f},(\widetilde{R}, \underset{\widetilde{B}}{\widetilde{f}}))$ is a $c \tau$-map approximating $f$ whose coloring for unbounded regions in $\mathbb{R}^{n} \backslash \widetilde{f}(S(\widetilde{f}))$ is in $\widetilde{R}$. We can show that this is well defined, and it defines a bordism ${ }^{3}$ invariant of smooth maps into $\mathbb{R}^{n}$. Also, it induces a cobordism invariant of the source manifold.

${ }^{3}$ Two smooth maps $f_{i}: M_{i} \rightarrow N$ for $i=0,1$ of closed manifolds are bordant if there exist a compact manifold $W$ whose boundary is the disjoint union of $M_{0}$ and $M_{1}$ and a smooth map $F: W \rightarrow N \times[0,1]$ such that $f_{i}=\left.F\right|_{M_{i}}: M_{i} \rightarrow N \times\{i\}$ for $i=0,1$; see [Conner and Floyd 1964] for details. 


\section{Stable maps of $n$-manifolds into 4-manifolds for $n=3$ and $n=5$}

Let us study the universal complex of the singular fibers of two-colored $C^{0}$ stable maps $^{4}$ of $n$-manifolds into 4-manifolds for $n=3$ and $n=5$.

4.1. Stable maps of 3-manifolds into 4-manifolds. By using the transversality theorem of Mather [1970], the following is proved.

Proposition 4.1. A proper smooth map $f: M \rightarrow N$ of a 3-manifold into a 4manifold is $C^{\infty}$ stable if and only if $f$ satisfies these conditions:

(i) (Local condition). For each $p \in M$, there exist local coordinates $(a, b, x)$ about $p$ and $(X, Y, Z, W)$ about $f(p) \in N$ such that

$(X \circ f, Y \circ f, Z \circ f, W \circ f)= \begin{cases}(a, b, x, 0) & \text { if } p \text { is a regular point, } \\ \left(a, b, a x, x^{2}\right) & \text { if } p \text { is a Whitney umbrella point } .\end{cases}$

(ii) (Global condition). For each $q \in f(M)$, the preimage $f^{-1}(q)$ consists of at most four points, and the germ $\left(f, f^{-1}(q)\right)$ is right-left equivalent to one of these seven germs:

(a) $f^{-1}(q)=\varnothing$, whose fiber we call $\varnothing$;

(b) a single immersion, whose fiber we call I;

(c) the normal crossing of two immersion germs, whose fiber we call 2I;

(d) the normal crossing of three immersion germs, whose fiber we call 3I;

(e) the map germ corresponding to a Whitney umbrella point, whose fiber we call $W U$;

(f) the normal crossing of four immersion germs, whose fiber we call 4I;

(g) the transverse crossing of a Whitney umbrella germ and an immersion germ, whose fiber we call $W U \pitchfork I$.

Remark 4.2. Since $(3,4)$ is in the nice range in the sense of Mather [1971], the characterization of $C^{\infty}$ stable maps of 3-manifolds into 4-manifolds gives the characterization of $C^{0}$ stable maps.

Consider the set $\mathscr{C} \mathscr{S}_{\mathrm{pr}}^{0}(3,4)$ of all fibers of proper two-colored stable maps of 3-manifolds into 4-manifolds. The set of all $C^{\infty}$ stable maps is open and dense in $C^{\infty}(M, N)$ with respect to the Whitney $C^{\infty}$ topology if $\operatorname{dim} M=3, \operatorname{dim} N=4$,

${ }^{4}$ A map $f: M \rightarrow N$ is $C^{\infty}$ stable (or $C^{0}$ stable) if the $C^{\infty} \mathscr{A}$-orbit (or $C^{0} \mathscr{A}$-orbit) of $f$ is open in $C^{\infty}(M, N)$ with respect to the Whitney $C^{\infty}$ topology, where $C^{\infty}(M, N)$ denotes the set of smooth maps $M \rightarrow N$. The $C^{\infty} \mathscr{A}$-orbit (or $C^{0} \mathscr{A}$-orbit) of $f$ is as follows: Let $\operatorname{Diff}(N)$ (or $\operatorname{Homeo}(N)$ ) denote the group of self-diffeomorphisms (or self-homeomorphisms) of $N$. The group $\operatorname{Diff}(M) \times \operatorname{Diff}(N)(\operatorname{or} \operatorname{Homeo}(M) \times \operatorname{Homeo}(N))$ acts on $C^{\infty}(M, N)$ by $(\Phi, \Psi) g=\Psi \circ g \circ \Phi^{-1}$, where $(\Phi, \Psi) \in \operatorname{Diff}(M) \times \operatorname{Diff}(N)($ or $(\Phi, \Psi) \in \operatorname{Homeo}(M) \times \operatorname{Homeo}(N))$ and $g \in C^{\infty}(M, N)$. The $C^{\infty} \mathscr{A}$-orbit (or $C^{0} \mathscr{A}$-orbit) of $f$ is the orbit through $f$ with respect to this action. Note that a proper $C^{\infty}$ stable map is also a Thom map. 

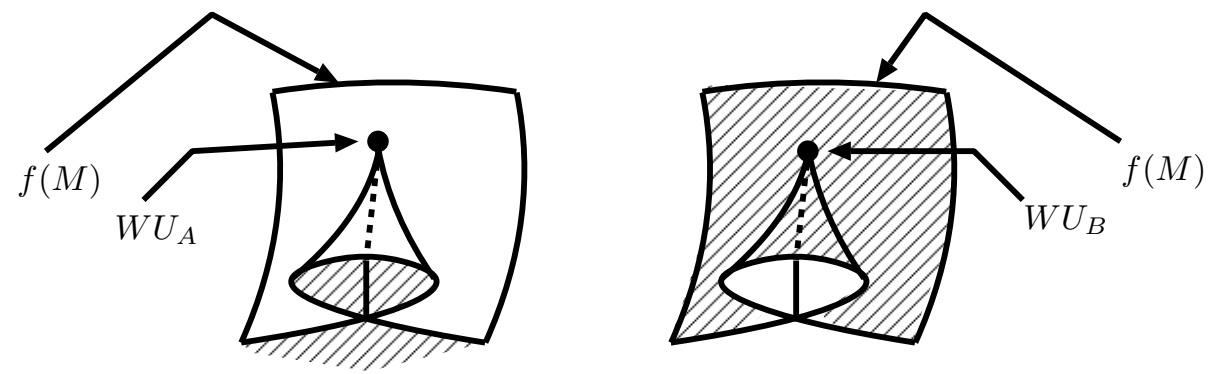

Figure 3. Two-colored $C^{0}$ equivalence classes of a Whitney umbrella point.

and $M$ is compact. Furthermore, if $N$ satisfies $H^{1}\left(N ; \mathbb{Z}_{2}\right)=0$, each smooth map is approximated by a two-colored stable map. $\mathscr{C} \mathscr{C}_{\mathrm{pr}}^{0}(3,4)$ is big enough.

Classify the elements of $\mathscr{C} \mathscr{S}_{\mathrm{pr}}^{0}(3,4)$ by the equivalence relation $c \rho_{3,4}^{0}$. Now let $(f,(R, B)): M \rightarrow N$ be a two-colored map whose fiber over $q_{0} \in N$ is $C^{0}$ equivalent to $\varnothing$. Define the $c \rho_{3,4}^{0}$ class of the fiber over $q_{0}$ to be of type $A$ if $q_{0}$ is in $R$; if $q_{0}$ is in $B$, call it of type $B$. Let the fiber of $(f,(R, B))$ over $q_{1} \in N$ be $C^{0}$ equivalent to $W U$. Then the $c \rho_{3,4}^{0}$ class of the fiber over $q_{1}$ is type $A$ if the inside the umbrella is in $R$ and is type $B$ if the outside of the umbrella is in $R$; see Figure 3. The $C^{0}$ classes of $\varnothing$ and $W U$ have two types $A$ and $B$ with respect to $c \rho_{3,4}^{0}$, whereas the other classes do not have such two types. We denote the two-colored $C^{0}$ equivalence class of $\varnothing$ and $W U$ of type $A$ by $\varnothing_{A}$ and $W U_{A}$, respectively, and adopt analogous notation for $B$ replacing $A$.

We obtain the universal complex $\left(C^{\kappa}\left(\mathscr{C} \mathcal{S}_{\mathrm{pr}}^{0}(3,4), c \rho_{3,4}^{0}\right), \delta_{\kappa}\right)_{\kappa}$, where the $C^{\kappa}$ are defined as

$$
\begin{array}{lll}
C^{0}=\left\langle\varnothing_{A}, \varnothing_{B}\right\rangle, & C^{2}=\langle 2 I\rangle, & C^{4}=\langle 4 I, W U \pitchfork I\rangle, \\
C^{1}=\langle I\rangle, & C^{3}=\left\langle 3 I, W U_{A}, W U_{B}\right\rangle, & C^{\kappa}=0 \text { otherwise. }
\end{array}
$$

The coboundary operations of the cochain complex are in Table 1.

Proposition 4.3. The cohomology groups of $\left(C^{\kappa}\left(\mathscr{C} \mathscr{Y}_{\mathrm{pr}}^{0}(3,4), c \rho_{3,4}^{0}\right), \delta_{\kappa}\right)_{\kappa}$ are as follows:

- $H^{0} \cong \mathbb{Z}_{2}$ generated by $\left[\varnothing_{A}+\varnothing_{B}\right]$;

- $H^{1}=\{0\}$;

- $H^{2}=\{0\}$;

- $H^{3} \cong \mathbb{Z}_{2}$ generated by $\left[W U_{A}+3 I\right] \equiv\left[W U_{B}+3 I\right]$.

Here $[*]$ denotes the cohomology class represented by the cycle $*$.

The third cohomology class of Proposition 4.3 implies Theorem 1.2. 


\begin{tabular}{llll}
\hline$\kappa$ & generator(s) & $\kappa$ & generator(s) \\
\hline 0 & $\delta_{0}\left(\varnothing_{A}\right)=0$, & 3 & $\delta_{3}(3 I)=W U \pitchfork I$, \\
& $\delta_{0}\left(\varnothing_{B}\right)=0$, & & $\delta_{3}\left(W U_{A}\right)=W U \pitchfork I$, \\
1 & $\delta_{1}(I)=0$, & & $\delta_{3}\left(W U_{B}\right)=W U \pitchfork I$, \\
2 & $\delta_{2}(2 I)=W U_{A}+W U_{B}$, & \\
\hline
\end{tabular}

Table 1. Generators for the coboundary groups of $\mathscr{b}\left(\mathscr{Y}_{\mathrm{pr}}^{0}(3,4), \rho_{3,4}^{0}\right)$.

Proof of Theorem 1.2. Denote the set of bordism classes of $p$-manifolds into a connected 3-manifold $N$ by $n_{p}(N)$. Note that we can choose the representative of the bordism class to be a two-colored stable map. On the other hand, we have

$$
n_{2}(N) \cong n_{2} \cong \mathbb{Z}_{2}=\left\langle w_{2}\right\rangle_{\mathbb{Z}_{2}},
$$

where $n_{2}$ denotes unoriented cobordism group of 2-manifolds and $w_{i}$ denotes the $i$-th Stiefel-Whitney class. See [Conner and Floyd 1964] and [Milnor and Stasheff 1974] for details of the first and second isomorphisms in (4-1), respectively. The representative of $n_{2}(N)$ can be chosen to be a two-colored map of a surface into a small neighborhood of a point in $N$; hence it can be considered as a map into $\mathbb{R}^{3}$. For $[c] \in H^{3}\left(\mathscr{C} \mathscr{S}_{\mathrm{pr}}^{0}(3,4), \rho_{3,4}^{0}\right)$, we have the map $[c]: n_{2}\left(\mathbb{R}^{3}\right) \rightarrow H^{3}\left(\mathbb{R}^{3}, \mathbb{Z}_{2}\right) \cong \mathbb{Z}_{2}$; see Remark 3.10. The immersion boy: $\mathbb{R} P^{2} \rightarrow \mathbb{R}^{3}$, constructed by Boy, represents the generator of $n_{2}\left(\mathbb{R}^{3}\right)$; the immersion has a triple point and no Whitney umbrella points, and so $[c]($ boy $)=1$; see [Francis 1987] for details. Therefore the map [c] is a projection.

4.2. Stable maps of 5-manifolds into 4-manifolds. The characterization of proper $C^{\infty}$ stable maps of 5-manifolds into 4-manifolds is well known; see [Saeki and Yamamoto 2006] or [Yamamoto 2007, Proposition 3.3] for details.

Remark 4.4. The characterization of $C^{\infty}$ stable maps of 5-manifolds into 4-manifolds also gives a characterization of $C^{0}$ stable maps of this kind, since $(5,4)$ is in the nice range in the sense of Mather; see [du Plessis and Wall 1995] for details.

In [Yamamoto 2007, Theorem 3.2], we obtained the list of $C^{\infty}$ classes of fibers of proper stable maps of 5-manifolds into 4-manifolds. For proper stable maps of closed 5-manifolds into 4-manifolds, the classification of the fibers with respect to the $C^{\infty}$ equivalence and that with respect to the $C^{0}$ equivalence coincide; see [Yamamoto 2007] for details.

Let us classify the fibers of proper two-colored stable maps of 5-manifolds into 4-manifolds with respect to the two-colored $C^{0}$ equivalence $c \rho_{5,4}^{0}$. (It is more sensitive than $\rho_{5,4}^{0}$.) Such equivalence splits some $C^{0}$ classes into two types $A$ and $B$.

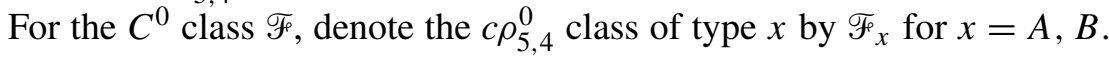



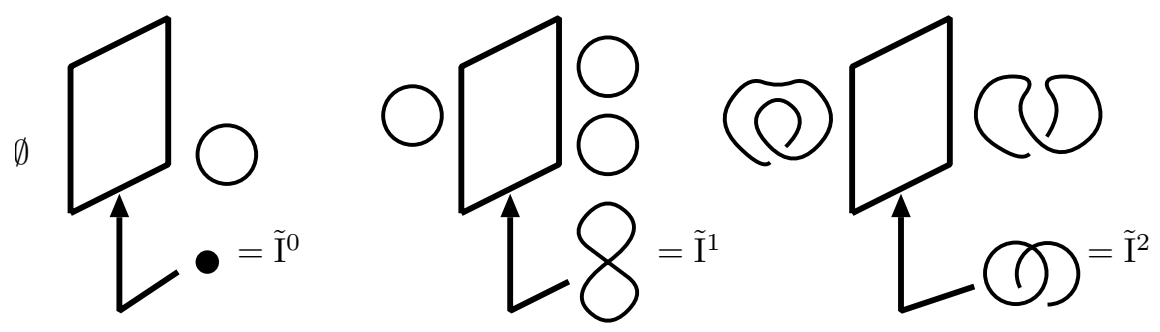

Figure 4. The fibers nearby $\tilde{\mathrm{I}}^{i}$ for $i=0,1,2$.

For each proper map $f: M \rightarrow N$ of an $n$-manifold into an (n-1)-manifold, each regular fiber is a disjoint union of trivial $S^{1}$ bundles, and the number of circle components in the inverse image is constant on each region in $N \backslash f(S(f))$. Assign each region the number of circles in the inverse image of a point in the region.

Let $(f,(R, B)): M \rightarrow N$ be a proper two-colored stable map of a 5-manifold into a 4-manifold.

Let 0 be a $C^{0}$ class of codimension zero, and let the fiber over $q_{0} \in N$ be $C^{0}$ equivalent to 0 . Say the $c \rho_{5,4}^{0}$ class of the fiber over $q$ is type $A$ if $q_{0}$ is in $R$, and say it is of type $B$ if $q_{0}$ is in $B$.

Let $\mathscr{E}$ be a $C^{0}$ class of codimension one, and let the fiber over $q_{1} \in N$ be $C^{0}$ equivalent to $\mathscr{E}$. Locally, $\mathscr{E}(f)$ is adjacent to two regions in $N \backslash f(S(f))$; see Figure 4. If $\mathscr{E}$ is $\tilde{\mathrm{I}}^{0}$ or $\tilde{\mathrm{I}}^{1}$, the difference between the associated numbers of such regions is one. Then say the $c \rho_{5,4}^{0}$ class of the fiber over $q_{1}$ is type $A$ if the region having larger associated number is in $R$, and adopt like language for $B$ replacing both $A$ and $R$. If $\mathscr{E}=\tilde{\mathrm{I}}^{2}$, the difference between the associated numbers of such regions is zero; see Figure 4 . The $C^{0}$ class of $\tilde{\mathrm{I}}^{2}$ does not have two types with respect to $c \rho_{5,4}^{0}$.

Let $\mathscr{F}$ be a $C^{0}$ class of codimension two, and let the fiber over $q_{2} \in N$ be $C^{0}$ equivalent to $\mathscr{F}$. Locally, $\mathscr{F}(f)$ is adjacent to four regions in $N \backslash f(S(f))$ unless $\mathscr{F}$ is $\widetilde{\mathrm{II}}^{a}$. Let $\mathscr{F}=\widetilde{\mathrm{II}}^{0,0}, \widetilde{\mathrm{II}}^{0,1}, \widetilde{\mathrm{II}}^{1,1}, \widetilde{\mathrm{II}}^{3}$, or $\widetilde{\mathrm{II}}^{5}$. Define the $c \rho_{5,4}^{0}$ class of the fiber over $q_{2}$ to be of type $A$ if the two of such regions in $R$ have the same associated number, and define the class to be of type $B$ otherwise; see Figure 5. In Figures 5, 6 , and 7 , the numbers attached to the regions are the associated numbers when the number of circle components in the inverse image of the center is zero. plus $5 \mathrm{pt}$

Let $\mathscr{F}=\widetilde{\mathrm{II}}^{4}$. Define the $c \rho_{5,4}^{0}$ class of the fiber over $q_{2}$ is type $A$ (or $B$ ) if two of such regions having the larger associated number are in $R$ (or $B$ ); see Figure 6. By a similar argument to the cases of $\tilde{\mathrm{I}}^{0}$ and $\tilde{\mathrm{I}}^{1}$, the $\widetilde{\mathrm{II}}^{a}$ have two types. In this way, the $C^{0}$ classes $\widetilde{\mathrm{II}}^{0,0}, \widetilde{\mathrm{II}}^{0,1}, \widetilde{\mathrm{II}}^{1,1}, \widetilde{\mathrm{II}}^{3}, \widetilde{\mathrm{I}}^{4}$, and $\widetilde{\mathrm{II}}^{a}$ have two types with respect to $c \rho_{5,4}^{0}$. However, the other $C^{0}$ classes of codimension two do not have two types; see Figure 7 for example. 

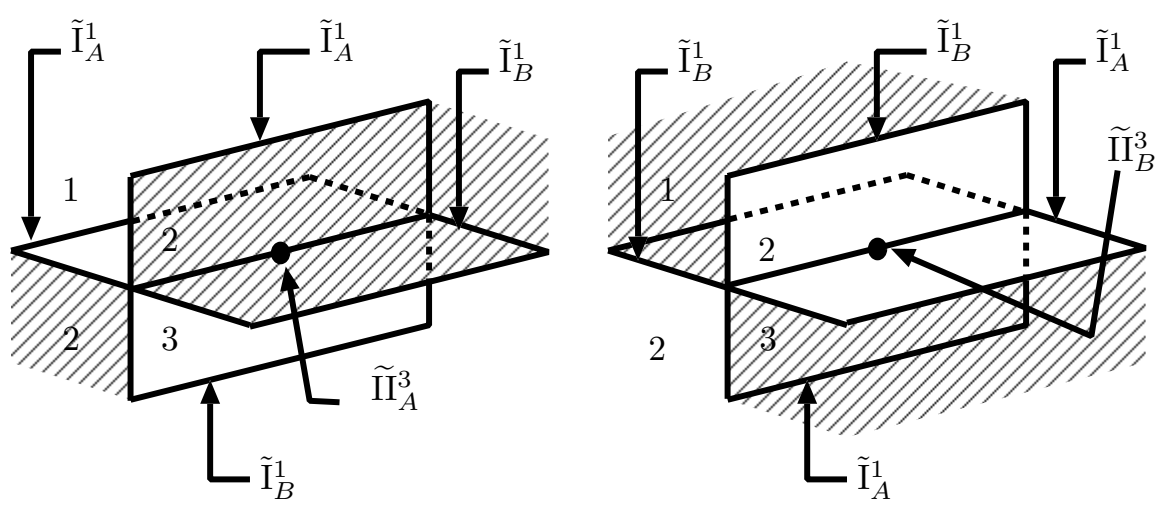

Figure 5. Types $A$ and $B$ for $\widetilde{I I}^{3}$.
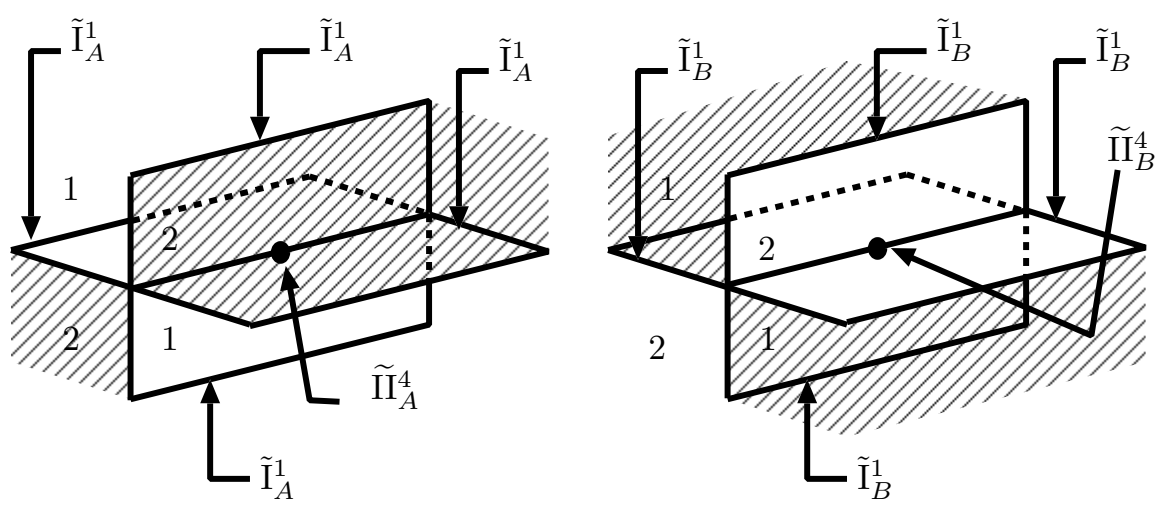

Figure 6. Types $A$ and $B$ for $\widetilde{I I}^{4}$.
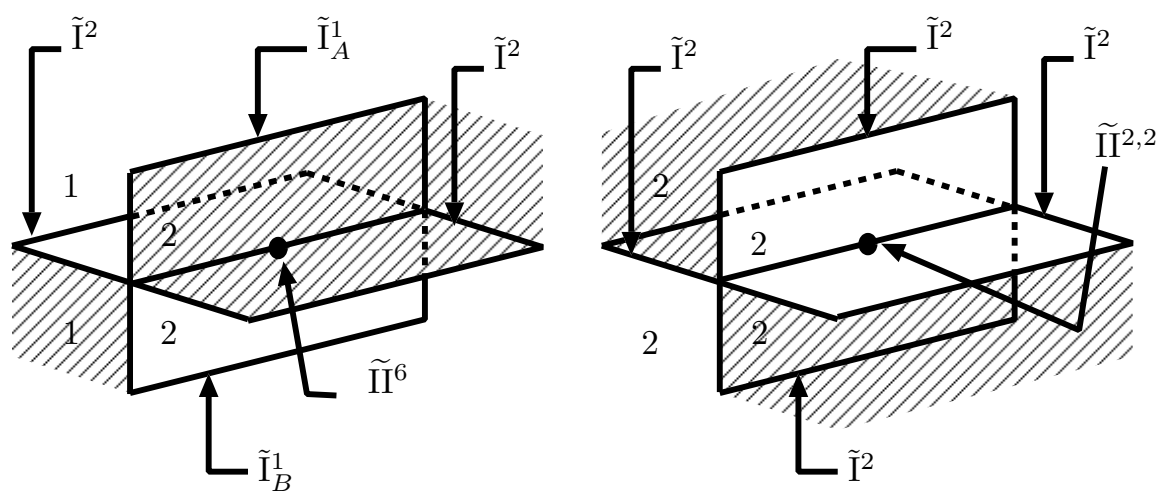

Figure 7. Codimension 2 strata that cannot be divided into two types. 
[Yamamoto 2006b] shows that, in the case $\kappa=3$, the $C^{0}$ classes

$$
\begin{aligned}
& \widetilde{\mathrm{III}}^{0,0,0}, \quad \widetilde{\mathrm{III}}^{0,0,1}, \quad \widetilde{\mathrm{III}}^{0,1,1}, \quad \widetilde{\mathrm{III}}^{1,1,1}, \\
& \tilde{\mathrm{III}}^{0,3}, \quad \widetilde{\mathrm{III}}^{0,4}, \quad \tilde{\mathrm{III}}^{0,5}, \quad \tilde{\mathrm{III}}^{1,3}, \quad \tilde{\mathrm{III}}^{1,4}, \quad \widetilde{\mathrm{III}}^{1,5}, \\
& \tilde{\mathrm{III}}^{8}, \quad \widetilde{\mathrm{III}}^{9}, \quad \widetilde{\mathrm{III}}^{10}, \quad \widetilde{\mathrm{III}}^{11}, \quad \widetilde{\mathrm{III}}^{12}, \quad \widetilde{\mathrm{III}}^{13}, \quad \widetilde{\mathrm{III}}^{15}, \quad \widetilde{\mathrm{III}}^{17}, \quad \widetilde{\mathrm{III}}^{21}, \\
& \widetilde{\mathrm{III}}^{0, a}, \quad \widetilde{\mathrm{III}}^{1, a}, \quad \widetilde{\mathrm{III}}^{b}, \quad \tilde{\mathrm{III}}^{d}, \quad \widetilde{\mathrm{III}}^{e}, \quad \tilde{\mathrm{III}}^{f}, \quad \tilde{\mathrm{III}}^{g}
\end{aligned}
$$

have two types with respect to $c \rho_{5,4}^{0}$; in the case $\kappa=4$, the classes with two types are

$$
\widetilde{\mathrm{IV}}^{0,0,0,0}, \quad \widetilde{\mathrm{IV}}^{0,0,0,1}, \quad \widetilde{\mathrm{IV}}^{0,0,1,1}, \quad \widetilde{\mathrm{IV}}^{0,1,1,1}, \quad \widetilde{\mathrm{IV}}^{1,1,1,1},
$$

$\widetilde{\mathrm{IV}}^{0,0,3}, \widetilde{\mathrm{IV}}^{0,0,4}, \widetilde{\mathrm{IV}}^{0,0,5}, \widetilde{\mathrm{IV}}^{0,1,3}, \widetilde{\mathrm{IV}}^{0,1,4}, \widetilde{\mathrm{IV}}^{0,1,5}, \widetilde{\mathrm{IV}}^{1,1,3}, \widetilde{\mathrm{IV}}^{1,1,4}, \widetilde{\mathrm{IV}}^{1,1,5}$,

$$
\tilde{\mathrm{IV}}^{0,0, a}, \quad \tilde{\mathrm{IV}}^{0,1, a}, \quad \tilde{\mathrm{IV}}^{1,1, a},
$$

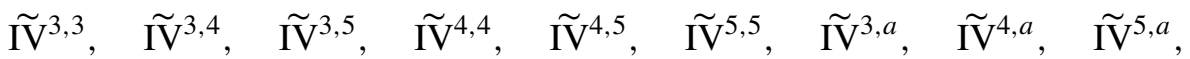
$\widetilde{\mathrm{IV}}^{0,8}, \widetilde{\mathrm{IV}}^{0,9}, \widetilde{\mathrm{IV}}^{0,10}, \widetilde{\mathrm{IV}}^{0,11}, \widetilde{\mathrm{IV}}^{0,12}, \widetilde{\mathrm{IV}}^{0,13}, \widetilde{\mathrm{IV}}^{0,15}, \widetilde{\mathrm{IV}}^{0,17}, \widetilde{\mathrm{IV}}^{0,21}$,

$\widetilde{\mathrm{IV}}^{1,8}, \tilde{\mathrm{IV}}^{1,9}, \widetilde{\mathrm{IV}}^{1,10}, \widetilde{\mathrm{IV}}^{1,11}, \widetilde{\mathrm{IV}}^{1,12}, \widetilde{\mathrm{IV}}^{1,13}, \widetilde{\mathrm{IV}}^{1,15}, \widetilde{\mathrm{IV}}^{1,17}, \widetilde{\mathrm{IV}}^{1,21}$, $\widetilde{\mathrm{IV}}^{0, b}, \widetilde{\mathrm{IV}}^{1, b}, \widetilde{\mathrm{IV}}^{0, d}, \widetilde{\mathrm{IV}}^{1, d}, \tilde{\mathrm{IV}}^{0, e}, \widetilde{\mathrm{IV}}^{1, e}, \widetilde{\mathrm{IV}}^{0, f}, \widetilde{\mathrm{IV}}^{1, f}, \widetilde{\mathrm{IV}}^{0, g}, \widetilde{\mathrm{IV}}^{1, g}, \widetilde{\mathrm{IV}}^{a, a}$,

$$
\widetilde{\mathrm{IV}}^{*} \text { for } *=27, \ldots, 40 \text {, }
$$

$\widetilde{I V}^{43}, \widetilde{I V}^{45}, \widetilde{I V}^{46}, \widetilde{I V}^{47}, \widetilde{I V}^{49}, \widetilde{I V}^{50}, \widetilde{I V}^{54}, \widetilde{I V}^{55}, \widetilde{I V}^{56}, \widetilde{I V}^{58}, \widetilde{I V}^{59}, \widetilde{I V}^{60}$, $\widetilde{I V}^{62}, \widetilde{I V}^{66}, \widetilde{I V}^{67}, \widetilde{I V}^{68}, \widetilde{I V}^{70}, \widetilde{I V}^{71}, \widetilde{I V}^{72}, \widetilde{I V}^{74}, \widetilde{I V}^{76}, \widetilde{I V}^{77}, \widetilde{I V}^{86}, \widetilde{I V}^{90}$, $\tilde{\mathrm{IV}}^{h}, \widetilde{\mathrm{IV}}^{i}, \tilde{\mathrm{IV}}^{j}, \widetilde{\mathrm{IV}}^{k}, \widetilde{\mathrm{IV}}^{q}, \tilde{\mathrm{IV}}^{r}, \widetilde{\mathrm{IV}}^{s}, \widetilde{\mathrm{IV}}^{t}, \tilde{\mathrm{IV}}^{u}, \widetilde{\mathrm{IV}}^{v}, \tilde{\mathrm{IV}}^{z}$,

$$
\tilde{\mathrm{IV}}^{\alpha}, \widetilde{\mathrm{IV}}^{\beta}, \widetilde{\mathrm{IV}}^{\gamma}, \widetilde{\mathrm{IV}}^{\delta}, \widetilde{\mathrm{IV}}^{\varepsilon}, \widetilde{\mathrm{IV}}^{\zeta}, \widetilde{\mathrm{IV}}^{\eta}
$$

The other $C^{0}$ classes of codimension three and four do not have two types.

Consider the set $\mathscr{C} \mathscr{S}_{\mathrm{pr}}^{0}(5,4)$ consisting of all fibers of proper two-colored stable maps of 5-manifolds into 4-manifolds. $\mathscr{C}_{\mathrm{pr}}^{0}(5,4)$ is big enough as well as the set $\mathscr{C} \mathscr{Y}_{\mathrm{pr}}^{0}(3,4)$.

Definition 4.5. Two fibers of proper Thom maps of $n$-manifolds into $(n-1)$-manifolds (for $n \geq 2$ ) are two-colored $C^{0}$ equivalent modulo $m$ circle components if one of them is two-colored $C^{0}$ equivalent to a disjoint union of the other one and $\mathrm{lm}$ copies of the fiber of the trivial $S^{1}$ bundle for some nonnegative integer $l$. Denote this equivalence relation by $c \rho_{n, n-1}^{0}(m)$.

We obtain the cochain complex

$$
\mathscr{C}\left(\mathscr{C} \mathscr{Y}_{\mathrm{pr}}^{0}(5,4), c \rho_{5,4}^{0}(2)\right)=\left(C^{\kappa}\left(\mathscr{C} \mathscr{Y}_{\mathrm{pr}}^{0}(5,4), c \rho_{5,4}^{0}(2)\right), \delta_{\kappa}\right)_{\kappa} .
$$

$C^{\kappa}\left(\mathscr{6} \mathcal{Y}_{\mathrm{pr}}^{0}(5,4), c \rho_{5,4}^{0}(2)\right)$ consists of all the $c \rho_{5,4}^{0}(2)$ classes of the fibers of codimension $\kappa$ in the list of [Yamamoto 2006a, Theorem 3.2]. The coboundaries are 
given in the following lists of equations. In these, the symbol $\mathscr{F}_{o}\left(\right.$ or $\mathscr{F}_{e}$ ) denotes the $c \rho_{5,4}^{0}(2)$ class of $\mathscr{F}_{F}$ such that the number of circle components of $\mathscr{F}$ is odd (or even). Denote $\mathscr{F}_{o}+\mathscr{F}_{e}$ by $\mathscr{F}$. The item $\delta_{K}\left(\mathscr{F}_{e}\right)$ can be obtained by interchanging $\mathscr{G}_{o}$ with $\mathscr{G}_{e}$ in the item $\delta_{\kappa}\left(\mathscr{F}_{e}\right)$ : if $\delta_{\kappa}\left(\mathscr{F}_{o}\right)=\mathscr{G}_{o}+\cdots$, then $\delta_{\kappa}\left(\mathscr{F}_{e}\right)=\mathscr{G}_{e}+\cdots$. We remark that $\delta_{K}\left(\gamma_{\kappa}(\mathscr{F})\right)=\gamma_{K}\left(\delta_{\kappa}(\mathscr{F})\right)$. We begin with the case $\kappa=0$, where

$$
\delta_{0}\left(0_{A, o}\right)=\tilde{\mathrm{I}}_{B, e}^{0}+\tilde{\mathrm{I}}_{A, e}^{1}+\tilde{\mathrm{I}}_{B, o}^{1}+\tilde{\mathrm{I}}_{e}^{2} .
$$

Next, the generators for $\kappa=1$ are

$$
\begin{aligned}
\delta_{1}\left(\tilde{\mathrm{I}}_{A, o}^{0}\right) & =\widetilde{\mathrm{II}}_{A, o}^{0,1}+\widetilde{\mathrm{II}}_{B, o}^{0,1}+\widetilde{\mathrm{I}}_{e}^{0,2}+\widetilde{\mathrm{II}}_{A, e}^{a}, \\
\delta_{1}\left(\widetilde{\mathrm{I}}_{A, o}^{1}\right) & =\widetilde{\mathrm{II}}_{A, o}^{0,1}+\widetilde{\mathrm{II}}_{B, e}^{0,1}+\widetilde{\mathrm{II}}_{e}^{1,2}+\widetilde{\mathrm{I}}_{A, o}^{a}, \\
\delta_{1}\left(\tilde{\mathrm{I}}_{o}^{2}\right) & =\widetilde{\mathrm{II}}^{0,2}+\widetilde{\mathrm{II}}^{1,2}+\widetilde{\mathrm{II}}^{6} .
\end{aligned}
$$

For $\kappa=2$, the generators are

$$
\begin{aligned}
& \delta_{2}\left(\widetilde{\mathrm{II}}_{A, o}^{0,0}\right)=\widetilde{\mathrm{III}}_{A, e}^{0,0,0}+\widetilde{\mathrm{III}}_{B, o}^{0,0,0}+\widetilde{\mathrm{III}}_{A, o}^{0,0,1}+\widetilde{\mathrm{III}}_{B, e}^{0,0,1}+\widetilde{\mathrm{II}}_{e}^{0,0,2}+\widetilde{\mathrm{III}}_{A, e}^{0, a}+\widetilde{\mathrm{II}}_{B, o}^{d}, \\
& \delta_{2}\left(\widetilde{\mathrm{II}}_{A, o}^{0,1}\right)=\widetilde{\mathrm{II}}_{A, o}^{0,1,1}+\widetilde{\mathrm{II}}_{B, e}^{0,1,1}+\widetilde{\mathrm{III}}_{e}^{0,1,2}+\widetilde{\mathrm{III}}_{o}^{0,6}+\widetilde{\mathrm{III}}_{A, o}^{0, a}+\widetilde{\mathrm{III}}_{A, e}^{1, a}+\widetilde{\mathrm{II}}_{A, o}^{b} \text {, } \\
& \delta_{2}\left(\widetilde{\mathrm{II}}_{A, o}^{1,1}\right)=\widetilde{\mathrm{III}}_{A, e}^{0,1,1}+\widetilde{\mathrm{III}}_{B, o}^{0,1,1}+\widetilde{\mathrm{III}}_{A, o}^{1,1,1}+\widetilde{\mathrm{II}}_{B, e}^{1,1,1}+\widetilde{\mathrm{II}}_{e}^{1,1,2}+\widetilde{\mathrm{III}}_{o}^{1,6}+\widetilde{\mathrm{III}}_{A, o}^{1, a}+\widetilde{\mathrm{III}}_{A, o}^{8} \text {, } \\
& \delta_{2}\left(\widetilde{\mathrm{II}}_{o}^{0,2}\right)=\widetilde{\mathrm{II}}^{0,1,2}+\widetilde{\mathrm{III}}^{0,6}+\widetilde{\mathrm{III}}_{e}^{2, a} \text {, } \\
& \delta_{2}\left(\widetilde{\mathrm{II}}_{o}^{1,2}\right)=\widetilde{\mathrm{III}}^{0,1,2}+\widetilde{\mathrm{III}}^{1,6}+\widetilde{\mathrm{III}}_{o}^{14}+\widetilde{\mathrm{III}}_{o}^{2, a}, \\
& \delta_{2}\left(\widetilde{\mathrm{II}}_{o}^{2,2}\right)=\widetilde{\mathrm{III}}^{0,2,2}+\widetilde{\mathrm{III}}^{1,2,2}+\widetilde{\mathrm{II}}^{2,6}+\widetilde{\mathrm{III}}_{o}^{20}, \\
& \delta_{2}\left(\widetilde{\mathrm{II}}_{A, o}^{3}\right)=\widetilde{\mathrm{II}}_{A, e}^{0,3}+\widetilde{\mathrm{II}}_{B, o}^{0,3}+\widetilde{\mathrm{II}}_{A, o}^{1,3}+\widetilde{\mathrm{II}}_{B, e}^{1,3}+\widetilde{\mathrm{II}}_{e}^{2,3}+\widetilde{\mathrm{II}}_{B, o}^{8}+\widetilde{\mathrm{II}}_{A, e}^{9}+\widetilde{\mathrm{II}}_{B, o}^{9}+\widetilde{\mathrm{II}}_{A, o}^{11} \\
& +\widetilde{\mathrm{III}}_{o}^{14}+\widetilde{\mathrm{II}}_{B, o}^{17}+\widetilde{\mathrm{III}}_{o}^{23}+\widetilde{\mathrm{II}}_{o}^{24}+\widetilde{\mathrm{III}}_{A, o}^{b}+\widetilde{\mathrm{II}}_{B, o}^{f}, \\
& \delta_{2}\left(\widetilde{\mathrm{II}}_{A, o}^{4}\right)=\widetilde{\mathrm{III}}_{A, e}^{0,4}+\widetilde{\mathrm{III}}_{B, o}^{0,4}+\widetilde{\mathrm{III}}_{A, o}^{1,4}+\widetilde{\mathrm{III}}_{B, e}^{1,4}+\widetilde{\mathrm{II}}_{e}^{2,4}+\widetilde{\mathrm{II}}_{A, o}^{10}+\widetilde{\mathrm{III}}_{B, e}^{10}+\widetilde{\mathrm{II}}_{A, o}^{11}+\widetilde{\mathrm{II}}_{B, o}^{13}
\end{aligned}
$$$$
+\widetilde{\mathrm{III}}_{A, o}^{21}+\widetilde{\mathrm{III}}_{A, o}^{e},
$$$$
\delta_{2}\left(\widetilde{\mathrm{II}}_{A, o}^{5}\right)=\widetilde{\mathrm{III}}_{A, o}^{0,5}+\widetilde{\mathrm{III}}_{B, e}^{0,5}+\widetilde{\mathrm{III}}_{A, e}^{1,5}+\widetilde{\mathrm{III}}_{B, o}^{1,5}+\widetilde{\mathrm{III}}_{A, e}^{15}+\widetilde{\mathrm{III}}_{A, o}^{17}+\widetilde{\mathrm{III}}_{A, o}^{21}+\widetilde{\mathrm{III}}_{B, o}^{g},
$$$$
\delta_{2}\left(\widetilde{\mathrm{II}}_{o}^{6}\right)=\widetilde{\mathrm{III}}^{0,6}+\widetilde{\mathrm{III}}^{1,6}+\widetilde{\mathrm{II}}_{e}^{14}+\widetilde{\mathrm{III}}_{o}^{c} \text {, }
$$$$
\delta_{2}\left(\widetilde{\mathrm{II}}_{o}^{7}\right)=\widetilde{\mathrm{III}}^{0,7}+\widetilde{\mathrm{III}}^{1,7}+\widetilde{\mathrm{II}}_{o}^{13}+\widetilde{\mathrm{III}}^{18}+\widetilde{\mathrm{II}}^{19}+\widetilde{\mathrm{II}}_{o}^{20}
$$$$
\delta_{2}\left(\widetilde{\mathrm{II}}_{A, o}^{a}\right)=\widetilde{\mathrm{III}}_{A, o}^{0, a}+\widetilde{\mathrm{III}}_{B, e}^{0, a}+\widetilde{\mathrm{III}}_{A, e}^{1, a}+\widetilde{\mathrm{III}}_{B, o}^{1, a}+\widetilde{\mathrm{II}}_{A, o}^{b}+\widetilde{\mathrm{II}}_{A, e}^{b} .
$$

For $\kappa=3$, the generators are

$$
\begin{aligned}
& \delta_{3}\left(\widetilde{\mathrm{III}}_{A, o}^{0,0,0}\right)= \widetilde{\mathrm{IV}}_{B, o}^{0,0,0,1}+\widetilde{\mathrm{IV}}_{A, e}^{0,0,0,1}+\widetilde{\mathrm{IV}}_{e}^{0,0,0,2}+\widetilde{\mathrm{IV}}_{A, e}^{0,0, a}+\widetilde{\mathrm{IV}}_{A, o}^{0, d}, \\
& \delta_{3}\left(\widetilde{\mathrm{III}}_{A, o}^{0,0,1}\right)=\widetilde{\mathrm{IV}}_{A, o}^{0,0,0,1}+\widetilde{\mathrm{IV}}_{B, e}^{0,0,0,1}+\widetilde{\mathrm{IV}}_{e}^{0,0,1,2}+\widetilde{\mathrm{IV}}_{o}^{0,0,6}+\widetilde{\mathrm{IV}}_{A, o}^{0,0, a}+\widetilde{\mathrm{IV}}_{A, e}^{0,1, a} \\
&+\widetilde{\mathrm{IV}}_{A, o}^{0, b}+\widetilde{\mathrm{IV}}_{A, o}^{1, d},
\end{aligned}
$$




$$
\begin{aligned}
& \delta_{3}\left(\widetilde{\mathrm{III}}_{A, o}^{0,1,1}\right)=\widetilde{\mathrm{IV}}_{B, o}^{0,1,1,1}+\widetilde{\mathrm{IV}}_{A, e}^{0,1,1,1}+\widetilde{\mathrm{IV}}_{e}^{0,1,1,2}+\widetilde{\mathrm{IV}}_{o}^{0,1,6} \\
& +\widetilde{\mathrm{IV}}_{A, o}^{0,1, a}+\widetilde{\mathrm{IV}}_{A, e}^{1,1, a}+\tilde{\mathrm{IV}}_{B, o}^{0,8}+\widetilde{\mathrm{IV}}_{A, o}^{1, b} \\
& \delta_{3}\left(\widetilde{\mathrm{III}}_{A, o}^{1,1,1}\right)=\widetilde{\mathrm{IV}}_{A, o}^{0,1,1,1}+\widetilde{\mathrm{IV}}_{B, e}^{0,1,1,1}+\widetilde{\mathrm{IV}}_{e}^{1,1,1,2}+\widetilde{\mathrm{IV}}_{o}^{1,1,6}+\widetilde{\mathrm{IV}}_{A, o}^{1,1, a}+\widetilde{\mathrm{IV}}_{B, o}^{1,8} \text {, } \\
& \delta_{3}\left(\widetilde{\mathrm{II}}_{A, o}^{0,3}\right)=\widetilde{\mathrm{IV}}_{B, o}^{0,1,3}+\widetilde{\mathrm{IV}}_{A, e}^{0,1,3}+\widetilde{\mathrm{IV}}_{e}^{0,2,3}+\widetilde{\mathrm{IV}}_{A, o}^{0,8}+\widetilde{\mathrm{IV}}_{A, o}^{0,9}+\widetilde{\mathrm{IV}}_{B, e}^{0,9}+\widetilde{\mathrm{IV}}_{A, o}^{0,11}+\widetilde{\mathrm{IV}}_{o}^{0,14} \\
& +\tilde{\mathrm{IV}}_{B, o}^{0,17}+\tilde{\mathrm{IV}}_{o}^{0,23}+\tilde{\mathrm{IV}}_{o}^{0,24}+\tilde{\mathrm{IV}}_{A, e}^{3, a}+\tilde{\mathrm{IV}}_{A, o}^{0, b}+\tilde{\mathrm{IV}}_{A, o}^{0, f}+\tilde{\mathrm{IV}}_{A, o}^{h}+\tilde{\mathrm{IV}}_{A, o}^{i}, \\
& \delta_{3}\left(\widetilde{\mathrm{III}}_{A, o}^{1,3}\right)=\widetilde{\mathrm{IV}}_{A, o}^{0,1,3}+\widetilde{\mathrm{IV}}_{B, e}^{0,1,3}+\widetilde{\mathrm{IV}}_{e}^{1,2,3}+\widetilde{\mathrm{IV}}_{A, o}^{1,8}+\widetilde{\mathrm{IV}}_{A, o}^{1,9}+\widetilde{\mathrm{IV}}_{B, e}^{1,9}+\widetilde{\mathrm{IV}}_{A, o}^{1,11}+\widetilde{\mathrm{IV}}_{o}^{1,14} \\
& +\widetilde{\mathrm{IV}}_{A, o}^{1,17}+\tilde{\mathrm{IV}}_{o}^{1,23}+\tilde{\mathrm{IV}}_{o}^{1,24}+\tilde{\mathrm{IV}}_{o}^{3,6}+\tilde{\mathrm{IV}}_{A, o}^{3, a}+\tilde{\mathrm{IV}}_{A, o}^{1, b}+\tilde{\mathrm{IV}}_{A, o}^{1, f}+\tilde{\mathrm{IV}}_{B, o}^{29}, \\
& \delta_{3}\left(\widetilde{\mathrm{III}}_{A, o}^{0,4}\right)=\widetilde{\mathrm{IV}}_{B, o}^{0,1,4}+\widetilde{\mathrm{IV}}_{A, e}^{0,1,4}+\widetilde{\mathrm{IV}}_{e}^{0,2,4}+\widetilde{\mathrm{IV}}_{A, o}^{0,10}+\widetilde{\mathrm{IV}}_{B, e}^{0,10}+\widetilde{\mathrm{IV}}_{A, o}^{0,11}+\widetilde{\mathrm{IV}}_{A, o}^{0,13} \\
& +\widetilde{\mathrm{IV}}_{B, o}^{0,21}+\tilde{\mathrm{IV}}_{o}^{0,22}+\widetilde{\mathrm{IV}}_{A, e}^{4, a}+\tilde{\mathrm{IV}}_{A, o}^{0, e}+\tilde{\mathrm{IV}}_{A, o}^{j} \\
& \delta_{3}\left(\widetilde{\mathrm{III}}_{A, o}^{1,4}\right)=\widetilde{\mathrm{IV}}_{A, o}^{0,1,4}+\widetilde{\mathrm{IV}}_{B, e}^{0,1,4}+\widetilde{\mathrm{IV}}_{e}^{1,2,4}+\widetilde{\mathrm{IV}}_{A, o}^{1,10}+\widetilde{\mathrm{IV}}_{B, e}^{1,10}+\widetilde{\mathrm{IV}}_{A, o}^{1,11}+\widetilde{\mathrm{IV}}_{A, o}^{1,13} \\
& +\widetilde{\mathrm{IV}}_{B, o}^{1,21}+\widetilde{\mathrm{IV}}_{o}^{1,22}+\tilde{\mathrm{IV}}_{o}^{4,6}+\tilde{\mathrm{IV}}_{A, o}^{4, a}+\widetilde{\mathrm{IV}}_{A, o}^{1, e}+\tilde{\mathrm{IV}}_{B, o}^{28} \\
& \delta_{3}\left(\widetilde{\mathrm{III}}_{A, o}^{8}\right)=\tilde{\mathrm{IV}}_{A, o}^{0,8}+\tilde{\mathrm{IV}}_{B, e}^{0,8}+\widetilde{\mathrm{IV}}_{B, o}^{1,8}+\tilde{\mathrm{IV}}_{A, e}^{1,8}+\tilde{\mathrm{IV}}_{e}^{2,8}+\tilde{\mathrm{IV}}_{o}^{41}+\tilde{\mathrm{IV}}_{o}^{42}+\tilde{\mathrm{IV}}_{o}^{44} \\
& +\widetilde{\mathrm{IV}}_{A, o}^{h}+\widetilde{\mathrm{IV}}_{A, o}^{s} \\
& \delta_{3}\left(\widetilde{I I I}_{A, o}^{9}\right)=\widetilde{\mathrm{IV}}_{A, o}^{0,9}+\widetilde{\mathrm{IV}}_{B, e}^{0,9}+\widetilde{\mathrm{IV}}_{B, o}^{1,9}+\widetilde{\mathrm{IV}}_{A, e}^{1,9}+\widetilde{\mathrm{IV}}_{e}^{2,9}+\widetilde{\mathrm{IV}}_{B, e}^{29}+\widetilde{\mathrm{IV}}_{A, o}^{31}
\end{aligned}
$$$$
+\widetilde{\mathrm{IV}}_{B, o}^{46}+\tilde{\mathrm{IV}}_{o}^{48}+\tilde{\mathrm{IV}}_{o}^{51}+\tilde{\mathrm{IV}}_{o}^{52}+\tilde{\mathrm{IV}}_{A, o}^{i}+\tilde{\mathrm{IV}}_{B, o}^{\gamma}
$$

$\delta_{3}\left(\widetilde{I I I}_{A, o}^{10}\right)=\tilde{\mathrm{IV}}_{B, o}^{0,10}+\widetilde{\mathrm{IV}}_{A, e}^{0,10}+\tilde{\mathrm{IV}}_{A, o}^{1,10}+\widetilde{\mathrm{IV}}_{B, e}^{1,10}+\tilde{\mathrm{IV}}_{e}^{2,10}+\tilde{\mathrm{IV}}_{A, e}^{28}+\widetilde{\mathrm{IV}}_{B, o}^{31}+\tilde{\mathrm{IV}}_{o}^{53}+\widetilde{\mathrm{IV}}_{B, o}^{54}$

$$
+\tilde{\mathrm{IV}}_{A, o}^{55}+\tilde{\mathrm{IV}}_{o}^{57}+\tilde{\mathrm{IV}}_{A, o}^{58}+\tilde{\mathrm{IV}}_{B, o}^{59}+\tilde{\mathrm{IV}}_{o}^{61}+\tilde{\mathrm{IV}}_{o}^{63}+\tilde{\mathrm{IV}}_{o}^{64}+\tilde{\mathrm{IV}}_{B, o}^{j}+\tilde{\mathrm{IV}}_{B, o}^{r}
$$

$\delta_{3}\left(\widetilde{\mathrm{III}}_{A, o}^{11}\right)=\tilde{\mathrm{IV}}_{B, o}^{0,11}+\widetilde{\mathrm{IV}}_{A, e}^{0,11}+\widetilde{\mathrm{IV}}_{A, o}^{1,11}+\widetilde{\mathrm{IV}}_{B, e}^{1,11}+\tilde{\mathrm{IV}}_{e}^{2,11}+\widetilde{\mathrm{IV}}_{B, o}^{31}+\tilde{\mathrm{IV}}_{A, e}^{31}$

$$
+\tilde{\mathrm{IV}}_{o}^{65}+\tilde{\mathrm{IV}}_{A, o}^{66}+\tilde{\mathrm{IV}}_{B, o}^{67}+\tilde{\mathrm{IV}}_{B, o}^{68}+\tilde{\mathrm{IV}}_{B, o}^{t}+\tilde{\mathrm{IV}}_{A, o}^{\gamma}
$$

$\delta_{3}\left(\widetilde{\mathrm{III}}_{A, o}^{12}\right)=\tilde{\mathrm{IV}}_{B, o}^{0,12}+\widetilde{\mathrm{IV}}_{A, e}^{0,12}+\widetilde{\mathrm{IV}}_{A, o}^{1,12}+\widetilde{\mathrm{IV}}_{B, e}^{1,12}+\tilde{\mathrm{IV}}_{e}^{2,12}+\widetilde{\mathrm{IV}}_{B, o}^{36}+\widetilde{\mathrm{IV}}_{A, e}^{36}$

$$
+\widetilde{\mathrm{IV}}_{o}^{69}+\widetilde{\mathrm{IV}}_{B, o}^{70}+\widetilde{\mathrm{IV}}_{A, o}^{71}
$$

$\delta_{3}\left(\widetilde{\mathrm{III}}_{A, o}^{0, a}\right)=\widetilde{\mathrm{IV}}_{A, o}^{0,1, a}+\widetilde{\mathrm{IV}}_{B, e}^{0,1, a}+\tilde{\mathrm{IV}}_{e}^{0,2, a}+\widetilde{\mathrm{IV}}_{A, o}^{0, b}+\widetilde{\mathrm{IV}}_{B, e}^{0, b}+\widetilde{\mathrm{IV}}_{o}^{0, c}+\widetilde{\mathrm{IV}}_{B, o}^{\alpha}$,

$\delta_{3}\left(\widetilde{\mathrm{II}}_{A, o}^{1, a}\right)=\widetilde{\mathrm{IV}}_{B, o}^{0,1, a}+\widetilde{\mathrm{IV}}_{A, e}^{0,1, a}+\widetilde{\mathrm{IV}}_{e}^{1,2, a}+\widetilde{\mathrm{IV}}_{o}^{6, a}+\widetilde{\mathrm{IV}}_{B, o}^{1, b}+\widetilde{\mathrm{IV}}_{A, e}^{1, b}+\widetilde{\mathrm{IV}}_{o}^{1, c}+\widetilde{\mathrm{IV}}_{A, o}^{h}+\widetilde{\mathrm{IV}}_{A, o}^{s}$, $\delta_{3}\left(\widetilde{\mathrm{III}}_{A, o}^{b}\right)=\widetilde{\mathrm{IV}}_{B, o}^{0, b}+\widetilde{\mathrm{IV}}_{A, e}^{0, b}+\widetilde{\mathrm{IV}}_{B, o}^{1, b}+\widetilde{\mathrm{IV}}_{A, e}^{1, b}+\widetilde{\mathrm{IV}}_{e}^{2, b}+\widetilde{\mathrm{IV}}_{B, e}^{h}+\widetilde{\mathrm{IV}}_{o}^{l}+\widetilde{\mathrm{IV}}_{o}^{m}+\tilde{\mathrm{IV}}_{o}^{n}$ $+\widetilde{\mathrm{IV}}_{A, o}^{\alpha}+\widetilde{\mathrm{IV}}_{B, e}^{s}$

$\delta_{3}\left(\widetilde{\mathrm{III}}_{A, o}^{d}\right)=\widetilde{\mathrm{IV}}_{A, o}^{0, d}+\widetilde{\mathrm{IV}}_{B, e}^{0, d}+\widetilde{\mathrm{IV}}_{B, o}^{1, d}+\tilde{\mathrm{IV}}_{A, e}^{1, d}+\widetilde{\mathrm{IV}}_{e}^{2, d}+\tilde{\mathrm{IV}}_{A, e}^{\alpha}$, $\delta_{3}\left(\widetilde{\mathrm{III}}_{A, o}^{e}\right)=\tilde{\mathrm{IV}}_{B, o}^{0, e}+\tilde{\mathrm{IV}}_{A, e}^{0, e}+\widetilde{\mathrm{IV}}_{A, o}^{1, e}+\tilde{\mathrm{IV}}_{B, e}^{1, e}+\tilde{\mathrm{IV}}_{e}^{2, e}+\tilde{\mathrm{IV}}_{B, o}^{r}+\tilde{\mathrm{IV}}_{A, e}^{r}$

$$
+\tilde{\mathrm{IV}}_{A, o}^{t}+\tilde{\mathrm{IV}}_{A, o}^{v}+\tilde{\mathrm{IV}}_{o}^{y}+\tilde{\mathrm{IV}}_{A, o}^{\gamma}+\tilde{\mathrm{IV}}_{A, o}^{\zeta}
$$


$\delta_{3}\left(\widetilde{\mathrm{III}}_{A, o}^{f}\right)=\widetilde{\mathrm{IV}}_{A, o}^{0, f}+\widetilde{\mathrm{IV}}_{B, e}^{0, f}+\widetilde{\mathrm{IV}}_{B, o}^{1, f}+\widetilde{\mathrm{IV}}_{A, e}^{1, f}+\widetilde{\mathrm{IV}}_{e}^{2, f}+\widetilde{\mathrm{IV}}_{A, o}^{s}+\widetilde{\mathrm{IV}}_{B, e}^{s}$

$$
+\tilde{\mathrm{IV}}_{B, o}^{t}+\tilde{\mathrm{IV}}_{A, o}^{u}+\tilde{\mathrm{IV}}_{o}^{x}+\tilde{\mathrm{IV}}_{A, o}^{\alpha}+\tilde{\mathrm{IV}}_{B, o}^{\gamma}
$$

$\delta_{3}\left(\widetilde{\mathrm{III}}_{o}^{0,0,2}\right)=\widetilde{\mathrm{IV}}^{0,0,0,2}+\widetilde{\mathrm{IV}}^{0,0,1,2}+\widetilde{\mathrm{IV}}^{0,0,6}+\widetilde{\mathrm{IV}}_{e}^{0,2, a}+\widetilde{\mathrm{IV}}_{o}^{0, c}+\widetilde{\mathrm{IV}}_{o}^{2, d}$,

$\delta_{3}\left(\widetilde{\mathrm{III}}_{o}^{0,2,2}\right)=\tilde{\mathrm{IV}}^{0,1,2,2}+\tilde{\mathrm{IV}}^{0,2,6}+\tilde{\mathrm{IV}}_{e}^{2,2, a}+\tilde{\mathrm{IV}}_{o}^{0,20}+\tilde{\mathrm{IV}}_{o}^{2, c}$,

$\delta_{3}\left(\widetilde{\mathrm{III}}_{o}^{1,1,2}\right)=\widetilde{\mathrm{IV}}^{1,1,1,2}+\widetilde{\mathrm{IV}}^{0,1,1,2}+\widetilde{\mathrm{IV}}^{1,1,6}+\widetilde{\mathrm{IV}}_{o}^{1,2, a}+\widetilde{\mathrm{IV}}_{o}^{1,14}+\widetilde{\mathrm{IV}}_{o}^{1,20}+\widetilde{\mathrm{IV}}_{o}^{2,8}$,

$\delta_{3}\left(\widetilde{\mathrm{III}}_{o}^{1,2,2}\right)=\widetilde{\mathrm{IV}}^{0,1,2,2}+\widetilde{\mathrm{IV}}^{1,2,6}+\widetilde{\mathrm{IV}}_{o}^{2,2, a}+\widetilde{\mathrm{IV}}_{o}^{2,14}+\widetilde{\mathrm{IV}}_{o}^{1,20}$,

$\delta_{3}\left(\widetilde{\mathrm{III}}_{o}^{0,1,2}\right)=\widetilde{\mathrm{IV}}^{0,1,6}+\widetilde{\mathrm{IV}}_{o}^{0,2, a}+\widetilde{\mathrm{IV}}_{e}^{1,2, a}+\widetilde{\mathrm{IV}}_{o}^{0,14}+\widetilde{\mathrm{IV}}_{o}^{2, b}+\widetilde{\mathrm{IV}}_{o}^{1, c}$,

$\delta_{3}\left(\widetilde{\mathrm{III}}_{o}^{2,2,2}\right)=\widetilde{\mathrm{IV}}^{0,2,2,2}+\widetilde{\mathrm{IV}}^{1,2,2,2}+\widetilde{\mathrm{IV}}^{2,2,6}+\widetilde{\mathrm{IV}}_{o}^{2,20}$,

$\delta_{3}\left(\widetilde{\mathrm{III}}_{A, o}^{0,5}\right)=\widetilde{\mathrm{IV}}_{B, o}^{0,1,5}+\widetilde{\mathrm{IV}}_{A, e}^{0,1,5}+\widetilde{\mathrm{IV}}_{o}^{0,2,5}+\widetilde{\mathrm{IV}}_{A, o}^{0,15}+\widetilde{\mathrm{IV}}_{B, e}^{0,15}+\widetilde{\mathrm{IV}}_{o}^{0,16}+\widetilde{\mathrm{IV}}_{B, o}^{0,17}$

$$
+\widetilde{\mathrm{IV}}_{A, o}^{0,21}+\widetilde{\mathrm{IV}}_{A, e}^{5, a}+\widetilde{\mathrm{IV}}_{A, o}^{0, g}+\tilde{\mathrm{IV}}_{A, o}^{k}
$$

$\delta_{3}\left(\widetilde{\mathrm{III}}_{o}^{0,6}\right)=\widetilde{\mathrm{IV}}^{0,1,6}+\widetilde{\mathrm{IV}}_{e}^{0,14}+\widetilde{\mathrm{IV}}_{e}^{6, a}+\widetilde{\mathrm{IV}}_{o}^{0, c}+\widetilde{\mathrm{IV}}_{o}^{l}+\widetilde{\mathrm{IV}}_{o}^{m}+\widetilde{\mathrm{IV}}_{o}^{n}$,

$\delta_{3}\left(\widetilde{\mathrm{III}}_{o}^{0,7}\right)=\widetilde{\mathrm{IV}}^{0,1,7}+\tilde{\mathrm{IV}}_{A, o}^{0,13}+\widetilde{\mathrm{IV}}_{B, o}^{0,13}+\widetilde{\mathrm{IV}}^{0,18}+\widetilde{\mathrm{IV}}^{0,19}+\tilde{\mathrm{IV}}_{o}^{0,20}+\widetilde{\mathrm{IV}}_{e}^{7, a}+\tilde{\mathrm{IV}}_{o}^{o}+\tilde{\mathrm{IV}}_{o}^{p}$, $\delta_{3}\left(\widetilde{\mathrm{III}}_{A, o}^{1,5}\right)=\widetilde{\mathrm{IV}}_{A, o}^{0,1,5}+\widetilde{\mathrm{IV}}_{B, e}^{0,1,5}+\widetilde{\mathrm{IV}}_{e}^{1,2,5}+\widetilde{\mathrm{IV}}_{A, o}^{1,15}+\widetilde{\mathrm{IV}}_{B, e}^{1,15}+\widetilde{\mathrm{IV}}_{o}^{1,16}+\widetilde{\mathrm{IV}}_{A, o}^{1,17}$

$$
+\widetilde{\mathrm{IV}}_{A, o}^{1,21}+\widetilde{\mathrm{IV}}_{o}^{5,6}+\widetilde{\mathrm{IV}}_{A, o}^{1, g}+\tilde{\mathrm{IV}}_{B, o}^{43},
$$

$\delta_{3}\left(\widetilde{\mathrm{III}}_{o}^{1,6}\right)=\widetilde{\mathrm{IV}}^{0,1,6}+\widetilde{\mathrm{IV}}_{e}^{1,14}+\widetilde{\mathrm{IV}}_{o}^{6, a}+\widetilde{\mathrm{IV}}_{o}^{1, c}+\widetilde{\mathrm{IV}}_{o}^{41}+\widetilde{\mathrm{IV}}_{e}^{42}+\widetilde{\mathrm{IV}}_{o}^{44}$,

$\delta_{3}\left(\widetilde{\mathrm{III}}_{o}^{1,7}\right)=\widetilde{\mathrm{IV}}^{0,1,7}+\widetilde{\mathrm{IV}}_{A, o}^{1,13}+\widetilde{\mathrm{IV}}_{B, o}^{1,13}+\widetilde{\mathrm{IV}}^{1,18}+\widetilde{\mathrm{IV}}^{1,19}+\widetilde{\mathrm{IV}}_{o}^{1,20}+\widetilde{\mathrm{IV}}_{o}^{7, a}+\widetilde{\mathrm{IV}}_{o}^{84}+\widetilde{\mathrm{IV}}_{o}^{85}$, $\delta_{3}\left(\widetilde{\mathrm{III}}_{o}^{2,3}\right)=\widetilde{\mathrm{IV}}^{0,2,3}+\widetilde{\mathrm{IV}}^{1,2,3}+\widetilde{\mathrm{IV}}_{o}^{2,8}+\widetilde{\mathrm{IV}}^{2,9}+\widetilde{\mathrm{IV}}_{o}^{2,11}+\widetilde{\mathrm{IV}}_{o}^{2,17}+\widetilde{\mathrm{IV}}^{3,6}$

$$
+\widetilde{\mathrm{IV}}_{e}^{2, b}+\widetilde{\mathrm{IV}}_{o}^{2, f}+\tilde{\mathrm{IV}}_{o}^{41}+\tilde{\mathrm{IV}}_{o}^{48},
$$

$\delta_{3}\left(\widetilde{\mathrm{III}}_{o}^{2,4}\right)=\widetilde{\mathrm{IV}}^{0,2,4}+\widetilde{\mathrm{IV}}^{1,2,4}+\widetilde{\mathrm{IV}}^{2,10}+\widetilde{\mathrm{IV}}_{o}^{2,11}+\widetilde{\mathrm{IV}}_{o}^{2,13}+\widetilde{\mathrm{IV}}_{o}^{2,21}+\widetilde{\mathrm{IV}}^{4,6}+\widetilde{\mathrm{IV}}_{o}^{2, e}+\widetilde{\mathrm{IV}}_{o}^{63}$, $\delta_{3}\left(\widetilde{\mathrm{III}}_{o}^{2,5}\right)=\widetilde{\mathrm{IV}}^{0,2,5}+\widetilde{\mathrm{IV}}^{1,2,5}+\widetilde{\mathrm{IV}}^{2,15}+\widetilde{\mathrm{IV}}_{o}^{2,17}+\widetilde{\mathrm{IV}}_{o}^{2,21}+\widetilde{\mathrm{IV}}^{5,6}+\widetilde{\mathrm{IV}}_{o}^{2, g}+\widetilde{\mathrm{IV}}_{o}^{82}$, $\delta_{3}\left(\widetilde{\mathrm{II}}_{o}^{2,6}\right)=\widetilde{\mathrm{IV}}^{0,2,6}+\widetilde{\mathrm{IV}}^{1,2,6}+\widetilde{\mathrm{IV}}_{e}^{2,14}+\widetilde{\mathrm{IV}}_{o}^{2, c}+\widetilde{\mathrm{IV}}_{o}^{80}+\widetilde{\mathrm{IV}}_{o}^{81}$, $\delta_{3}\left(\widetilde{\mathrm{III}}_{o}^{2,7}\right)=\widetilde{\mathrm{IV}}^{0,2,7}+\widetilde{\mathrm{IV}}^{1,2,7}+\widetilde{\mathrm{IV}}_{o}^{2,13}+\widetilde{\mathrm{IV}}^{2,18}+\widetilde{\mathrm{IV}}^{2,19}+\widetilde{\mathrm{IV}}_{o}^{2,20}+\widetilde{\mathrm{IV}}^{6,7}+\widetilde{\mathrm{IV}}_{o}^{104}+\widetilde{\mathrm{IV}}_{o}^{109}$, $\delta_{3}\left(\widetilde{\mathrm{III}}_{A, o}^{13}\right)=\widetilde{\mathrm{IV}}_{A, o}^{0,13}+\widetilde{\mathrm{IV}}_{B, e}^{0,13}+\tilde{\mathrm{IV}}_{B, o}^{1,13}+\widetilde{\mathrm{IV}}_{A, e}^{1,13}+\tilde{\mathrm{IV}}_{e}^{2,13}+\widetilde{\mathrm{IV}}_{A, o}^{54}+\widetilde{\mathrm{IV}}_{B, e}^{54}$ $+\tilde{\mathrm{IV}}_{A, o}^{59}+\tilde{\mathrm{IV}}_{B, e}^{59}+\tilde{\mathrm{IV}}_{A, o}^{70}+\tilde{\mathrm{IV}}_{B, e}^{70}+\tilde{\mathrm{IV}}_{o}^{73}+\tilde{\mathrm{IV}}_{o}^{75}$ $\delta_{3}\left(\widetilde{\mathrm{II}}_{o}^{14}\right)=\tilde{\mathrm{IV}}^{0,14}+\widetilde{\mathrm{IV}}^{1,14}+\widetilde{\mathrm{IV}}^{41}+\widetilde{\mathrm{IV}}^{42}+\widetilde{\mathrm{IV}}^{44}+\tilde{\mathrm{IV}}_{o}^{l}+\widetilde{\mathrm{IV}}_{o}^{x}$, $\delta_{3}\left(\widetilde{\mathrm{III}}_{A, o}^{15}\right)=\tilde{\mathrm{IV}}_{B, o}^{0,15}+\widetilde{\mathrm{IV}}_{A, e}^{0,15}+\widetilde{\mathrm{IV}}_{A, o}^{1,15}+\widetilde{\mathrm{IV}}_{B, e}^{1,15}+\widetilde{\mathrm{IV}}_{e}^{2,15}+\widetilde{\mathrm{IV}}_{A, e}^{43}+\widetilde{\mathrm{IV}}_{A, o}^{46}+\widetilde{\mathrm{IV}}_{B, o}^{58}$ $+\tilde{\mathrm{IV}}_{o}^{78}+\tilde{\mathrm{IV}}_{o}^{82}+\tilde{\mathrm{IV}}_{o}^{87}+\tilde{\mathrm{IV}}_{o}^{88}+\tilde{\mathrm{IV}}_{o}^{89}+\tilde{\mathrm{IV}}_{B, o}^{k}+\tilde{\mathrm{IV}}_{B, o}^{z}+\tilde{\mathrm{IV}}_{B, o}^{\zeta}+\tilde{\mathrm{IV}}_{B, o}^{55}$ $\delta_{3}\left(\widetilde{\mathrm{III}}_{o}^{16}\right)=\widetilde{\mathrm{IV}}^{0,16}+\widetilde{\mathrm{IV}}^{1,16}+\widetilde{\mathrm{IV}}_{o}^{6,7}+\widetilde{\mathrm{IV}}^{78}+\widetilde{\mathrm{IV}}_{o}^{79}+\widetilde{\mathrm{IV}}_{o}^{82}+\widetilde{\mathrm{IV}}^{87}+\widetilde{\mathrm{IV}}^{88}$

$$
+\widetilde{\mathrm{IV}}^{89}+\widetilde{\mathrm{IV}}_{o}^{91}+\widetilde{\mathrm{IV}}_{o}^{94}+\tilde{\mathrm{IV}}_{o}^{w}
$$




$$
\begin{aligned}
\delta_{3}\left(\widetilde{\mathrm{III}}_{A, o}^{17}\right)=\widetilde{\mathrm{IV}}_{A, o}^{0,17}+\widetilde{\mathrm{IV}}_{B, e}^{0,17}+\widetilde{\mathrm{IV}}_{B, o}^{1,17}+\tilde{\mathrm{IV}}_{A, e}^{1,17}+\widetilde{\mathrm{IV}}_{e}^{2,17}+\widetilde{\mathrm{IV}}_{B, o}^{4,6}+\widetilde{\mathrm{IV}}_{A, e}^{4,6} \\
+\widetilde{\mathrm{IV}}_{A, o}^{66}+\widetilde{\mathrm{IV}}_{A, o}^{67}+\widetilde{\mathrm{IV}}_{A, o}^{68}+\widetilde{\mathrm{IV}}_{o}^{79}+\widetilde{\mathrm{IV}}_{A, o}^{u},
\end{aligned}
$$

$\delta_{3}\left(\widetilde{\mathrm{III}}_{o}^{18}\right)=\tilde{\mathrm{IV}}^{0,18}+\tilde{\mathrm{IV}}^{1,18}+\widetilde{\mathrm{IV}}_{A, o}^{59}+\widetilde{\mathrm{IV}}_{B, o}^{59}+\widetilde{\mathrm{IV}}_{o}^{78}+\widetilde{\mathrm{IV}}_{o}^{81}+\widetilde{\mathrm{IV}}_{e}^{84}+\tilde{\mathrm{IV}}_{o}^{91}+\tilde{\mathrm{IV}}^{101}+\tilde{\mathrm{IV}}_{o}^{o}$, $\delta_{3}\left(\widetilde{\mathrm{III}}_{o}^{19}\right)=\tilde{\mathrm{IV}}^{0,19}+\tilde{\mathrm{IV}}^{1,19}+\widetilde{\mathrm{IV}}_{A, o}^{54}+\tilde{\mathrm{IV}}_{B, o}^{54}+\tilde{\mathrm{IV}}_{o}^{78}+\tilde{\mathrm{IV}}_{o}^{80}+\tilde{\mathrm{IV}}_{e}^{85}+\tilde{\mathrm{IV}}_{o}^{91}+\tilde{\mathrm{IV}}^{101}+\tilde{\mathrm{IV}}_{o}^{p}$ $\delta_{3}\left(\widetilde{\mathrm{III}}_{o}^{20}\right)=\widetilde{\mathrm{IV}}^{0,20}+\widetilde{\mathrm{IV}}^{1,20}+\widetilde{\mathrm{IV}}^{80}+\widetilde{\mathrm{IV}}^{81}$, $\delta_{3}\left(\widetilde{\mathrm{II}}_{o}^{21}\right)=\tilde{\mathrm{IV}}_{A, o}^{0,21}+\tilde{\mathrm{IV}}_{B, e}^{0,21}+\widetilde{\mathrm{IV}}_{B, o}^{1,21}+\tilde{\mathrm{IV}}_{A, e}^{1,21}+\tilde{\mathrm{IV}}_{e}^{2,21}+\tilde{\mathrm{IV}}_{A, o}^{55}+\tilde{\mathrm{IV}}_{B, e}^{55}+\tilde{\mathrm{IV}}_{A, o}^{58}$

$$
+\tilde{\mathrm{IV}}_{B, e}^{58}+\tilde{\mathrm{IV}}_{A, o}^{66}+\tilde{\mathrm{IV}}_{A, o}^{68}+\widetilde{\mathrm{IV}}_{o}^{91}+\tilde{\mathrm{IV}}_{o}^{94}+\tilde{\mathrm{IV}}_{A, o}^{v}+\widetilde{\mathrm{IV}}_{A, o}^{\zeta}+\tilde{\mathrm{IV}}_{B, o}^{67}
$$

$\delta_{3}\left(\widetilde{\mathrm{III}}_{o}^{22}\right)=\widetilde{\mathrm{IV}}^{0,22}+\widetilde{\mathrm{IV}}^{1,22}+\widetilde{\mathrm{IV}}^{53}+\widetilde{\mathrm{IV}}^{57}+\widetilde{\mathrm{IV}}^{61}+\widetilde{\mathrm{IV}}_{o}^{63}+\widetilde{\mathrm{IV}}^{64}+\widetilde{\mathrm{IV}}_{o}^{65}+\widetilde{\mathrm{IV}}_{A, o}^{68}$

$$
+\widetilde{\mathrm{IV}}_{B, o}^{68}+\widetilde{\mathrm{IV}}_{A, o}^{70}+\tilde{\mathrm{IV}}_{B, o}^{70}+\tilde{\mathrm{IV}}_{o}^{73}+\tilde{\mathrm{IV}}_{o}^{75}+\tilde{\mathrm{IV}}_{o}^{91}+\tilde{\mathrm{IV}}_{o}^{94}+\tilde{\mathrm{IV}}_{o}^{y}
$$

$\delta_{3}\left(\widetilde{\mathrm{II}}_{o}^{23}\right)=\widetilde{\mathrm{IV}}^{0,23}+\widetilde{\mathrm{IV}}^{1,23}+\tilde{\mathrm{IV}}_{e}^{42}+\widetilde{\mathrm{IV}}_{A, o}^{45}+\widetilde{\mathrm{IV}}_{B, o}^{45}+\tilde{\mathrm{IV}}_{A, o}^{55}+\widetilde{\mathrm{IV}}_{B, o}^{55}$

$$
+\widetilde{\mathrm{IV}}_{A, o}^{66}+\widetilde{\mathrm{IV}}_{B, o}^{66}+\tilde{\mathrm{IV}}_{o}^{n}
$$

$\delta_{3}\left(\widetilde{\mathrm{II}}_{o}^{24}\right)=\tilde{\mathrm{IV}}^{0,24}+\tilde{\mathrm{IV}}^{1,24}+\tilde{\mathrm{IV}}_{e}^{44}+\tilde{\mathrm{IV}}_{A, o}^{45}+\tilde{\mathrm{IV}}_{B, o}^{45}+\tilde{\mathrm{IV}}_{o}^{48}+\tilde{\mathrm{IV}}^{51}+\tilde{\mathrm{IV}}^{52}$

$$
+\widetilde{\mathrm{IV}}_{A, o}^{55}+\widetilde{\mathrm{IV}}_{B, o}^{55}+\widetilde{\mathrm{IV}}_{o}^{65}+\widetilde{\mathrm{IV}}_{o}^{79}+\tilde{\mathrm{IV}}_{o}^{m}
$$

$\delta_{3}\left(\widetilde{\mathrm{III}}_{o}^{25}\right)=\widetilde{\mathrm{IV}}^{0,25}+\widetilde{\mathrm{IV}}^{1,25}+\widetilde{\mathrm{IV}}_{A, o}^{71}+\widetilde{\mathrm{IV}}_{B, o}^{71}+\widetilde{\mathrm{IV}}_{o}^{75}+\widetilde{\mathrm{IV}}_{o}^{93}+\widetilde{\mathrm{IV}}^{99}+\widetilde{\mathrm{IV}}^{100}+\widetilde{\mathrm{IV}}_{o}^{104}$, $\delta_{3}\left(\widetilde{\mathrm{II}}_{o}^{26}\right)=\tilde{\mathrm{IV}}^{0,26}+\tilde{\mathrm{IV}}^{1,26}+\tilde{\mathrm{IV}}_{o}^{73}+\tilde{\mathrm{IV}}_{o}^{93}+\tilde{\mathrm{IV}}^{102}+\tilde{\mathrm{IV}}^{105}+\tilde{\mathrm{IV}}_{o}^{109}$,

$\delta_{3}\left(\widetilde{\mathrm{III}}_{o}^{2, a}\right)=\widetilde{\mathrm{IV}}^{0,2, a}+\widetilde{\mathrm{IV}}^{1,2, a}+\widetilde{\mathrm{IV}}^{6, a}+\widetilde{\mathrm{IV}}^{2, b}+\widetilde{\mathrm{IV}}_{o}^{l}+\widetilde{\mathrm{IV}}_{o}^{x}$,

$\delta_{3}\left(\widetilde{\mathrm{III}}_{o}^{c}\right)=\widetilde{\mathrm{IV}}^{0, c}+\widetilde{\mathrm{IV}}^{1, c}+\widetilde{\mathrm{IV}}_{o}^{l}+\widetilde{\mathrm{IV}}^{m}+\widetilde{\mathrm{IV}}^{n}+\widetilde{\mathrm{IV}}_{e}^{x}$,

$\delta_{3}\left(\widetilde{\mathrm{II}}_{o}^{g}\right)=\widetilde{\mathrm{IV}}_{B, o}^{0, g}+\widetilde{\mathrm{IV}}_{A, e}^{0, g}+\widetilde{\mathrm{IV}}_{A, o}^{1, g}+\widetilde{\mathrm{IV}}_{B, e}^{1, g}+\tilde{\mathrm{IV}}_{e}^{2, g}+\tilde{\mathrm{IV}}_{B, o}^{u}+\tilde{\mathrm{IV}}_{B, o}^{v}+\tilde{\mathrm{IV}}_{B, o}^{z}$

$$
+\widetilde{\mathrm{IV}}_{A, e}^{z}+\tilde{\mathrm{IV}}_{o}^{w}+\tilde{\mathrm{IV}}_{A, e}^{\zeta}
$$

Proposition 4.6. The cohomology groups of $\left(C^{\kappa}\left(\mathscr{C}_{\mathrm{pr}}^{0}(5,4), c \rho_{5,4}^{0}(2)\right), \delta_{\kappa}\right)_{\kappa}$ are as follows:

- $H^{0} \cong \mathbb{Z}_{2}$ generated by $\left[0_{A}+0_{B}\right]$;

- $H^{1} \cong \mathbb{Z}_{2} \oplus \mathbb{Z}_{2}$ generated by $\left[\tilde{\mathrm{I}}_{A, o}^{0}+\tilde{\mathrm{I}}_{B, o}^{0}+\tilde{\mathrm{I}}_{A, e}^{1}+\tilde{\mathrm{I}}_{B, e}^{1}\right] \equiv\left[\tilde{\mathrm{I}}_{A, e}^{0}+\tilde{\mathrm{I}}_{B, e}^{0}+\tilde{\mathrm{I}}_{A, o}^{1}+\tilde{\mathrm{I}}_{B, o}^{1}\right]$ and $\left[\tilde{\mathrm{I}}_{A}^{0}+\tilde{\mathrm{I}}_{A}^{1}+\tilde{\mathrm{I}}_{o}^{2}\right] \equiv\left[\tilde{\mathrm{I}}_{A}^{0}+\tilde{\mathrm{I}}_{A}^{1}+\tilde{\mathrm{I}}_{e}^{2}\right] \equiv\left[\tilde{\mathrm{I}}_{B}^{0}+\tilde{\mathrm{I}}_{B}^{1}+\tilde{\mathrm{I}}_{o}^{2}\right] \equiv\left[\tilde{\mathrm{I}}_{B}^{0}+\tilde{\mathrm{I}}_{B}^{1}+\tilde{\mathrm{I}}_{e}^{2}\right]$;

- $H^{2}=\{0\}$;

- $H^{3} \cong \mathbb{Z}_{2}$ generated by $\left[\widetilde{\widetilde{I I I}}^{2,2,2}+\widetilde{\mathrm{III}}^{2,7}+\widetilde{\mathrm{II}}_{A}^{12}+\widetilde{\mathrm{II}}_{\underline{B}}^{12}+\widetilde{\mathrm{III}}_{A, o}^{13}+\widetilde{\mathrm{III}}_{\underline{\sim}, e}^{13}+\widetilde{\mathrm{III}}^{25}+\right.$ $\left.\widetilde{\mathrm{III}}^{26}\right] \equiv\left[\widetilde{\mathrm{III}}^{2,2,2}+\widetilde{\mathrm{III}}^{2,7}+\widetilde{\mathrm{III}}_{A}^{12}+\widetilde{\mathrm{III}}_{B}^{12}+\widetilde{\mathrm{III}}_{A, e}^{13}+\widetilde{\mathrm{III}}_{B, o}^{13}+\widetilde{\mathrm{III}}^{25}+\widetilde{\mathrm{III}}^{26}\right]$.

where $[*]$ denotes the cohomology class represented by the cocycle $*$.

The third cohomology class of Proposition 4.6 implies Theorem 1.1. 
Proof of Theorem 1.1. By using arguments similar to those in the proof of Theorem 1.2 , the set of bordism classes of 4-manifolds into $N$ is

$$
n_{4}(N) \cong n_{4} \cong \mathbb{Z}_{2} \oplus \mathbb{Z}_{2}=\left\langle w_{4}\right\rangle_{\mathbb{Z}_{2}} \oplus\left\langle w_{1}^{4}\right\rangle_{\mathbb{Z}_{2}} .
$$

We can choose the representative of the bordism class by two-colored stable map into $\mathbb{R}^{3}$ as well. For $[c] \in H^{3}\left(\mathscr{C}_{\mathrm{pr}}^{0}(5,4), c \rho_{5,4}^{0}(2)\right)$, we get the map $[c]: n_{4}\left(\mathbb{R}^{3}\right) \rightarrow$ $H^{3}\left(\mathbb{R}^{3}, \mathbb{Z}_{2}\right) \cong \mathbb{Z}_{2}$; see Remark 3.10. One generator of $n_{4}\left(\mathbb{R}^{3}\right)$ is represented by the stable map $S_{a}: \mathbb{R} P^{2} \widetilde{\times} \mathbb{R} P^{2} \rightarrow \mathbb{R}^{3}$ constructed by Saeki [1992, Example 3.7]. $\left.S_{a}\right|_{S\left(S_{a}\right)}$ has 27 triple points. One is $\widetilde{I I}^{2,2,2}$, and the others are not any of $\widetilde{I I I}^{2,7}$, $\widetilde{\mathrm{III}}^{12}, \widetilde{\mathrm{III}}^{13}, \widetilde{\mathrm{III}}^{25}$, or $\widetilde{\mathrm{III}}^{26}$, and so $[c]\left(S_{a}\right)=1$. The other generator is represented by the stable map $K_{o}: \mathbb{C} P^{2} \rightarrow \mathbb{R}^{3}$ constructed by Kobayashi [1997]. $\left.K_{o}\right|_{S\left(K_{o}\right)}$ has two triple points. One is $\widetilde{\mathrm{III}}^{12}$ and the other is $\widetilde{\mathrm{III}}^{0,0,0}$, and so $[c]\left(K_{o}\right)=1$. Therefore, the map $[c]$ is the projection onto the first component.

Remark 4.7. The quotient cochain complex $\mathscr{C}\left(\mathscr{C} \mathscr{S}_{\mathrm{pr}}^{0}(5,4), c \rho_{5,4}^{0}(2)\right) / \gamma$ is isomorphic to the cochain complex $\mathscr{C}\left(\mathscr{S}_{\mathrm{pr}}^{0}(5,4), \rho_{5,4}^{0}(2)\right)$, where $\mathscr{Y}_{\mathrm{pr}}^{0}(5,4)$ is the set of all singular fibers of proper stable maps of 5-manifolds into 4-manifolds and $\rho_{5,4}^{0}(2)$ is the $C^{0}$ equivalence modulo two regular fiber components. $\mathscr{C}\left(\mathscr{P}_{\mathrm{pr}}^{0}(5,4), \rho_{5,4}^{0}(2)\right)$ also contains an interesting application; see [Yamamoto 2007] for details.

\section{Acknowledgments}

The author would like to thank adviser Go-o Ishikawa for helpful advice and encouragement; Osamu Saeki for helpful comments, discussions, and constant encouragement; Akiko Neriugawa for useful advice on English grammar and for support and encouragement; and finally the referee for careful reading and for many essential comments which improved the paper.

\section{References}

[Conner and Floyd 1964] P. E. Conner and E. E. Floyd, Differentiable periodic maps, Ergebnisse der Mathematik 33, Academic Press, Publishers, New York, 1964. MR 31 \#750 Zbl 0125.40103

[Francis 1987] G. K. Francis, A topological picturebook, Springer, New York, 1987. MR 88a:57002 Zbl 0612.57001

[Gibson et al. 1976] C. G. Gibson, K. Wirthmüller, A. A. du Plessis, and E. J. N. Looijenga, Topological stability of smooth mappings, Lecture Notes in Math. 552, Springer, Berlin, 1976. MR 55 \#9151 Zbl 0377.58006

[Ikegami and Saeki 2003] K. Ikegami and O. Saeki, "Cobordism group of Morse functions on surfaces”, J. Math. Soc. Japan 55:4 (2003), 1081-1094. MR 2004g:57046 Zbl 1046.57020

[Kalmár 2007] B. Kalmár, "Cobordism group of fold maps of oriented 3-manifolds into the plane", Acta Math. Hungar. 117:1-2 (2007), 1-25. MR 2356183

[Kobayashi 1997] M. Kobayashi, "Two stable maps of $\mathbf{C}^{2} \mathrm{P}$ into $\mathbf{R}^{3}$ ", Mem. College Ed. Akita Univ. Natur. Sci. 51 (1997), 5-12. MR 98g:57049 Zbl 0884.57026 
[Koschorke 1981] U. Koschorke, Vector fields and other vector bundle morphisms - a singularity approach, Lecture Notes in Math. 847, Springer, Berlin, 1981. MR 82i:57026 Zbl 0459.57016

[Mather 1970] J. N. Mather, "Stability of $C^{\infty}$ mappings. V. Transversality", Advances in Math. 4 (1970), 301-336. MR 43 \#1215c Zbl 0207.54303

[Mather 1971] J. N. Mather, "Stability of $C^{\infty}$ mappings. VI: The nice dimensions", pp. 207-253 in Proceedings of Liverpool Singularities Symposium, I (1969/70), Lecture Notes in Math. 192, Springer, Berlin, 1971. MR 45 \#2747 Zbl 0211.56105

[Milnor and Stasheff 1974] J. W. Milnor and J. D. Stasheff, Characteristic classes, Princeton University Press, NJ, 1974. Annals of Mathematics Studies, No. 76. MR 55 \#13428 Zbl 0298.57008

[du Plessis and Wall 1995] A. du Plessis and T. Wall, The geometry of topological stability, London Mathematical Society Monographs. New Series 9, The Clarendon Press Oxford University Press, New York, 1995. Oxford Science Publications. MR 97k:58024 Zbl 0870.57001

[Rimányi and Szúcs 1998] R. Rimányi and A. Szúcs, "Pontrjagin-Thom-type construction for maps with singularities”, Topology 37:6 (1998), 1177-1191. MR 99g:57031 Zbl 0924.57035

[Saeki 1992] O. Saeki, "Notes on the topology of folds", J. Math. Soc. Japan 44:3 (1992), 551-566. MR 93f:57037 Zbl 0764.57017

[Saeki 2004] O. Saeki, Topology of singular fibers of differentiable maps, Lecture Notes in Math. 1854, Springer, Berlin, 2004. MR 2005m:58085 Zbl 1072.57023

[Saeki and Yamamoto 2006] O. Saeki and T. Yamamoto, "Singular fibers of stable maps and signatures of 4-manifolds", Geom. Topol. 10 (2006), 359-399. MR 2007f:57058 Zbl 1107.57019

[Szúcs 1986] A. Szúcs, "Surfaces in $\mathbf{R}^{3}$ ”, Bull. London Math. Soc. 18 (1986), 60-66. MR 88a:57060 Zbl 0563.57015

[Thom 1954] R. Thom, "Quelques propriétés globales des variétés différentiables”, Comment. Math. Helv. 28 (1954), 17-86. MR 15,890a Zbl 0057.15502

[Yamamoto 2006a] T. Yamamoto, "Classification of singular fibres of stable maps of 4-manifolds into 3-manifolds and its applications”, J. Math. Soc. Japan 58 (2006), 721-742. MR 2007m:57035

[Yamamoto 2006b] T. Yamamoto, Cobordism invariants and the singular fibres of two colored maps, $\mathrm{PhD}$ thesis, Hokkaido University, 2006, Available at http://hd1.handle.net/2115/8465.

[Yamamoto 2007] T. Yamamoto, "Euler number formulas in terms of singular fibers of stable maps", pp. 427-457 in Real and complex singularities (Sydney, 2005), edited by A. Harris et al., World Sci. Publ., Hackensack, NJ, 2007. MR 2336698 Zbl 05179100

Received April 20, 2007. Revised November 7, 2007.

\section{TAKAHIRO YAMAMOTO}

FACULTY OF MATHEMATICS

KYUSHU UNIVERSITY

HAKOZAKI, FUKUOKA 812-8581

JAPAN

taku_chan@math.sci.hokudai.ac.jp 